\title{
2D Materials Based Optoelectronic Memory: Convergence of Electronic Memory and Optical Sensor
}

\author{
Feichi Zhou ${ }^{1}{ }^{1}$, Jiewei Chen ${ }^{1}$, Xiaoming Tao ${ }^{2}$, Xinran Wang ${ }^{3}$, and Yang Chai ${ }^{1}{ }^{1}$ \\ ${ }^{1}$ Department of Applied Physics, The Hong Kong Polytechnic University, Hong Kong \\ ${ }^{2}$ Institute of Textiles and Clothing, The Hong Kong Polytechnic University, Hong Kong \\ ${ }^{3}$ School of Electronic Science and Engineering, Nanjing University, Nanjing, China \\ Correspondence should be addressed to Yang Chai; ychai@polyu.edu.hk
}

Received 29 April 2019; Accepted 14 July 2019; Published 21 August 2019

Copyright (c) 2019 Feichi Zhou et al. Exclusive Licensee Science and Technology Review Publishing House. Distributed under a Creative Commons Attribution License (CC BY 4.0).

\begin{abstract}
The continuous development of electron devices towards the trend of "More than Moore" requires functional diversification that can collect data (sensors) and store (memories) and process (computing units) information. Considering the large occupation proportion of image data in both data center and edge devices, a device integration with optical sensing and data storage and processing is highly demanded for future energy-efficient and miniaturized electronic system. Two-dimensional (2D) materials and their heterostructures have exhibited broadband photoresponse and high photoresponsivity in the configuration of optical sensors and showed fast switching speed, multi-bit data storage, and large ON/OFF ratio in memory devices. In addition, its ultrathin body thickness and transfer process at low temperature allow 2D materials to be heterogeneously integrated with other existing materials system. In this paper, we overview the state-of-the-art optoelectronic random-access memories (ORAMs) based on 2D materials, as well as ORAM synaptic devices and their applications in neural network and image processing. The ORAM devices potentially enable direct storage/processing of sensory data from external environment. We also provide perspectives on possible directions of other neuromorphic sensor design (e.g., auditory and olfactory) based on 2D materials towards the future smart electronic systems for artificial intelligence.
\end{abstract}

\section{Introduction}

The advance of digital technology enables data storage and processing in binary form with high speed, accuracy and density. The development of computing hardware has been driven by Moore's law in the past few decades. However, the downward scaling trend is hitting the scaling limits according to the International Technology Roadmap for Semiconductors (ITRS) 2.0. Different from the strategy of device miniaturization involved in the digital components, a new trend of "More-than-Moore" (MtM) is highlighted in the ITRS 2.0, which is featured as a diversified function of data collection and information storage/ processing devices. The function diversification described in MtM can be considered as another form of "scaling down" for the electronic system, which includes noncomputing functions into the digital ones, enabling the interaction of digital devices with the outside world directly. Device integration of noncomputing (e.g., sensors and actuators) and digital components (e.g., memories and computing units) is one of the main approaches for function diversification of MtM. Considering the large occupation proportion of image data in both data center and edge devices, we will mainly discuss the integrations of optical sensors and digital devices (data storage and processing) in this review.

Optoelectronic random-access memories (ORAMs) offer great opportunities for multifunctional integration of optical sensing, data storage and processing into one single device. In contrast to traditional random-access memories (RAMs), optical excitation provides another method to manipulate the memory cell in ORAMs, which enables to store light information with an electronic readout at low programming voltages and is beneficial for the storage and transport of secured information. The combination of both optical stimuli and electrical readout voltage has potential for enlarging the ratio of low resistance state (LRS) and high resistance state (HRS), thus enabling multilevel data storage and improving data storage capacity. From another aspect, the optical trigger is employed in ORAM for the switching, bringing the benefits 


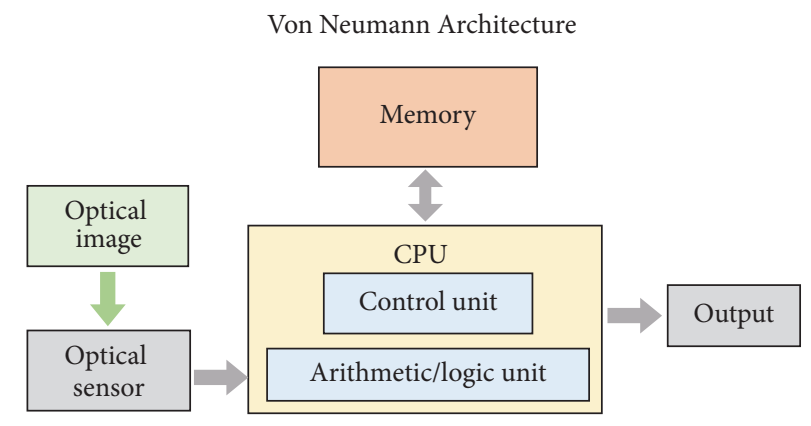

(a)

Novel Architecture based on ORAM

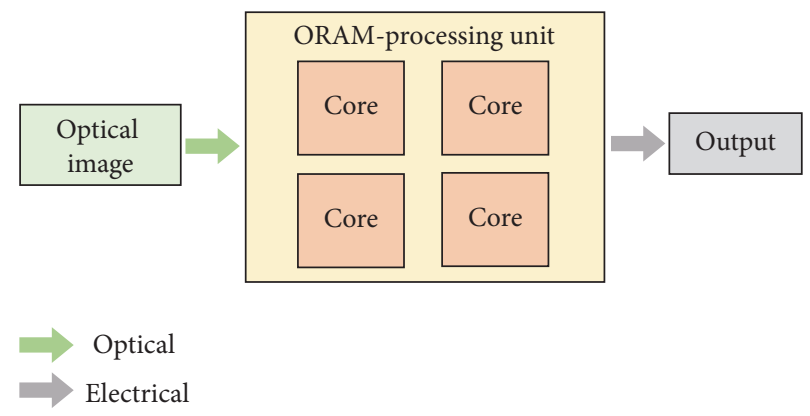

(b)

FIGURE 1: (a) Illustration of conventional Von Neumann architecture for image processing. (b) Novel computing architecture based on ORAM/ORAM synaptic devices for image processing.

of wide bandwidth and reduction of electrical loss during sensory data transmission [1-6]. In addition, it is promising to perform digital processing functions with light as an extra control parameter by combining both optical and electrical inputs as input signals, such as arithmetic and logic operations and coincidence detection $[4,7]$. Furthermore, the integration of optical sensors and memory devices in ORAMs provides possibilities in simplifying the image sensor circuitry.

In the conventional von Neumann computing architecture, memory and processing units are physically separated, which is inefficient in terms of energy consumption and data transmission speed. The human brain consists of a large collection of neurons connected through synapses, and deals with learning and processing complex cognition tasks (e.g., pattern recognition, and multiobject detection) with high energy efficiency. The power consumption in human brain is 5 orders magnitude lower than conventional computers [8]. Neuromorphic computing mimics the working schemes of information storage and processing like the human brain. The electronic memory synaptic devices are the most investigated and fundamental component in the hardware-based neuromorphic computing. The tunable resistance under continuous stimuli of voltage or current inputs is similar to the synaptic weight (generally determined as biological strength of the connection between two neurons). However, the conventional memory synaptic devices for neuromorphic computing are manipulated through the electrical method and are isolated from the sensors (e.g., visual, auditory or olfactory) [9-17]. ORAM synaptic devices integrate optical sensors and synaptic RAMs, which can respond to optical stimuli and exhibit light-tunable synaptic behaviors, including light-tunable short-term plasticity (STP), long-term plasticity (LTP), spike-timing-dependent plasticity (STDP), and spatiotemporal learning rule (STLR). The development of ORAM synaptic devices can provide potentials for bridging the gap between optical (visual) sensing and neuromorphic computing [18]. Figure 1 illustrates a comparison of the conventional Von Neumann architecture and a computing architecture based on the ORAM/ORAM synaptic devices for image storage and processing. The architecture based on ORAM/ORAM synaptic devices offer the possibilities of directly storing and processing the optical information, in contrast to the separated memory and processing unit and preliminary optical-to-electrical signal conversion for image processing in the conventional Von Neumann architecture.

Among different materials systems for ORAMs, twodimensional (2D) materials, such as graphene, transition metal dichalcogenides (TMDs) (including $\mathrm{MoS}_{2}, \mathrm{WS}_{2}, \mathrm{WSe}_{2}$, $\mathrm{MoSe}_{2}, \mathrm{MoTe}_{2}$, etc.) and black phosphorus, have exhibited unprecedented electrical and optical properties for the applications of soft optoelectronics and nonvolatile memories. In terms of the integration of optical sensing, data storage and processing, 2D materials and their heterostructures also exhibit promising potentials for building ORAMs with broadband photo response, excellent photoresponsivity [1925], fast switching speed, multibit data storage, large ON/OFF ratio and high energy efficiency [26-32]. More specifically, (1) 
the strong light-matter interactions and the gate tunability owing to the unique physical and structural properties in the ultrathin $2 \mathrm{D}$ materials benefit the construction of both volatile and nonvolatile ORAMs. (2) The large-surface-tovolume ratio in these $2 \mathrm{D}$ materials allows significant photoexcited charge trapping and further the ORAM design. (3) The mechanical flexibility and optical transparency of 2D materials are appropriate for the application of ORAMs in an artificial eye. The structure and physical properties of $2 \mathrm{D}$ materials have been overviewed in the existing reports [33-35]. In this paper, we mainly focused on the review and perspective of $2 \mathrm{D}$ materials for memory applications.

\section{2D Materials Based Nonvolatile Electronic Memory}

The $2 \mathrm{D}$ materials based electronic memory provides ideas for the design of $2 \mathrm{D}$ materials based ORAM structure. The working mechanisms of state-of-the-art ORAMs are generally based on charging trapping in three-terminal FET memory and light-tunable filament formation/rupture in two-terminal resistive random-access memories (RRAMs). In this part, we will mainly review two types of nonvolatile electronic memory based on 2D materials: floating gate memories and RRAMs.

$2 \mathrm{D}$ materials and their heterostructures have offered great practical advantages in the design of floating gate memory device with a large $\mathrm{ON} / \mathrm{OFF}$ ratio and long retention time. The application of $2 \mathrm{D}$ materials as the floating gate can effectively reduce the capacitive interference between the floating gate and drain electrode for high-capacity data storage. In early 2013, Bertolazzi et al. designed a nonvolatile floating gate memory with $\mathrm{MoS}_{2}$ as channel material, multilayer graphene as floating gate and charge trapping layer, and $\mathrm{HfO}_{2}$ as control gate (Figure 2(a)). As a positive control gate voltage is applied $(+18 \mathrm{~V})$, electrons tunnel from $\mathrm{MoS}_{2}$ channel to multilayer graphene floating gate through the $\mathrm{HfO}_{2}$ tunneling barrier. The electrons are stored in the floating gate, programming the device. A negative control gate voltage (-18 V) sweeps the electrons in the floating gate back to the channel material, erasing the device (Figure 2(b)). With this structure, the device exhibits nonvolatile switching characteristics with ON/OFF ratio of $10^{4}$ [36]. Later, Choi et al. demonstrated a similar floating gate memory with $\mathrm{MoS}_{2} / \mathrm{h}-\mathrm{BN} /$ graphene heterostructure with $\mathrm{h}-\mathrm{BN}$ as a tunneling barrier, showing a comparable ON/OFF ratio of $10^{4}$ [37]. By further tuning the h-BN thickness appropriately, two-terminal operation of the $\mathrm{MoS}_{2} / \mathrm{h}-\mathrm{BN} /$ graphene floating gate memory can be achieved (Figure 2(c)), which allows for higher integration density. A source-drain voltage of $-6 \mathrm{~V}$ programs the device to LRS state by driving the electrons to tunnel through the h-BN layer and be accumulated at graphene floating gate. A positive sourcedrain voltage of $6 \mathrm{~V}$ then is employed for erasing the device (Figure 2(d)). The device exhibits an extremely low OFF current of $10^{-14} \mathrm{~A}$, large ON/OFF ratio of up to $10^{9}$, and long retention time $\left(10^{4} \mathrm{~s}\right)$ [38]. As the operation speed in floating gate memories is usually relatively slow, a semi-floating gate memory based on van der Waals heterostructures was further developed as an effective device structure to achieve ultrahigh speed and long refresh time (Figure 2(e)). In the semi-floating gate memory, $\mathrm{WSe}_{2}$ is the channel material. $\mathrm{HfS}_{2}$ and h-BN serve as a floating gate and tunneling layer (drain region), respectively. The $\mathrm{WSe}_{2} / \mathrm{MoS}_{2}$ (source region) works as a $p$ $n$ junction switch. For the programming operation $\left(\mathrm{V}_{\mathrm{g}}=-5\right.$ $\left.\mathrm{V}, \mathrm{V}_{\mathrm{DS}}=0 \mathrm{~V}\right)$, the $p-n$ junction is switched on. The electrons in the $\mathrm{HfS}_{2}$ are transferred to the channel material through the h-BN tunneling layer (flash path) or the $\mathrm{WSe}_{2} / \mathrm{MoS}_{2} p-n$ junction (switch path). For the erasing operation $\left(\mathrm{V}_{\mathrm{g}}=5 \mathrm{~V}\right.$, $\mathrm{V}_{\mathrm{DS}}=0 \mathrm{~V}$ ), as the $p-n$ junction is switched off, electrons in the channel are transferred to the floating gate and combined with holes in the $\mathrm{HfS}_{2}$ layer. The fast writing speed and long refresh time in the device design originate from the fast charge injection through switch path and floating gate charge storage, respectively [28].

Although excellent nonvolatile properties can be achieved in the $2 \mathrm{D}$ nonvolatile floating gate memory, its three-terminal structure limits the integration density and requires large programming voltage. Thus, the two-terminal RRAMs are considered as promising candidates towards nonvolatile memory applications for the high integration density and low programming voltage. Two-terminal RRAMs based on 2D TMD monolayers have also been rationally designed for low operation voltage, including $\mathrm{MoS}_{2}, \mathrm{MoSe}_{2}, \mathrm{WS}_{2}$ and $\mathrm{WSe}_{2}$ [32]. The good thermal stability and well-defined interfaces of 2D heterostructures provide possibilities in overcoming the thermal stability issue in the conventional RRAMs. Wang et al. reported a robust $\mathrm{RRAM}$ based on graphene/ $\mathrm{MoS}_{2-\mathrm{x}} \mathrm{O}_{\mathrm{x}} /$ graphene heterostructure with excellent endurance up to $10^{7}$ switching cycles and high operating temperature of $340^{\circ} \mathrm{C}$ (Figures 3(a) and 3(c)) [29]. The high-temperature endurance of the device originates from the atomically sharp interfaces even at elevated temperatures, avoiding the undesirable ion migrations (Figure 3(b)). The 2D materials RRAMs also show promising potentials in constructing artificial synapses with low power consumption for energy-efficient neuromorphic computing. Shi et al. reported a 2D material RRAM synaptic device with metal/h-BN/metal structure (Figure 3(d)) based on the switching mechanism of formation/rupture of conductive filament $(\sim 15 \mathrm{~nm})$. The RRAM synaptic device exhibits both nonvolatile and volatile switching behaviors, as well as the tunable synaptic behaviors (Figures 3(e) and 3(f)). The low power consumption $(0.1 \mathrm{fW}$ in standby and $600 \mathrm{pW}$ per transition) and fast switching speed (10 ns) implicate the potential applications for brain-like computing [39].

\section{2D Materials Based Nonvolatile ORAM}

The conventional 2D materials based RRAM is usually manipulated by electrical triggers. As $2 \mathrm{D}$ materials have exhibited a broad response from ultraviolet to infrared light with high responsibility or fast response speed, it is possible to integrate the optical sensing and data storage together by rationally designing device structures, which is potential for the various applications, such as image sensors 


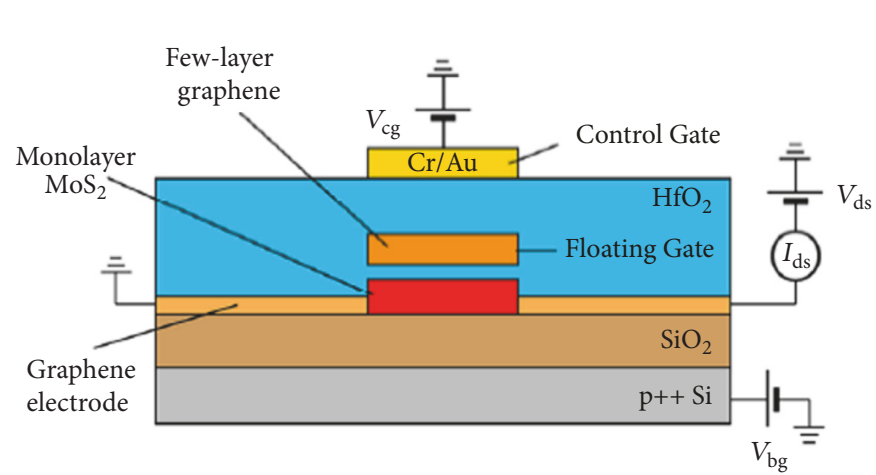

(a)

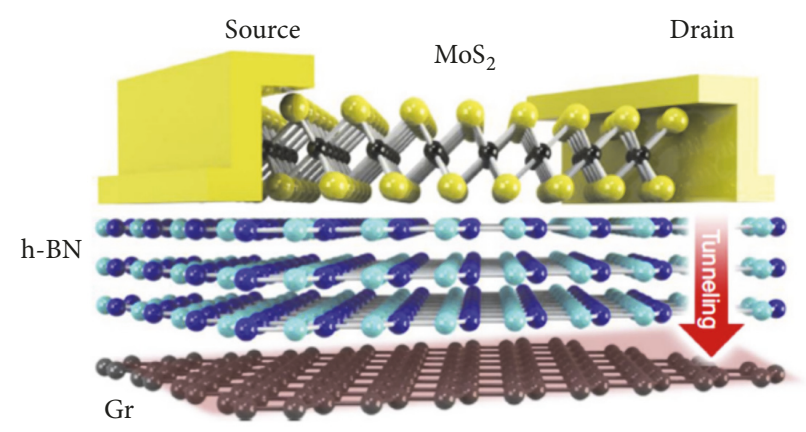

(c)

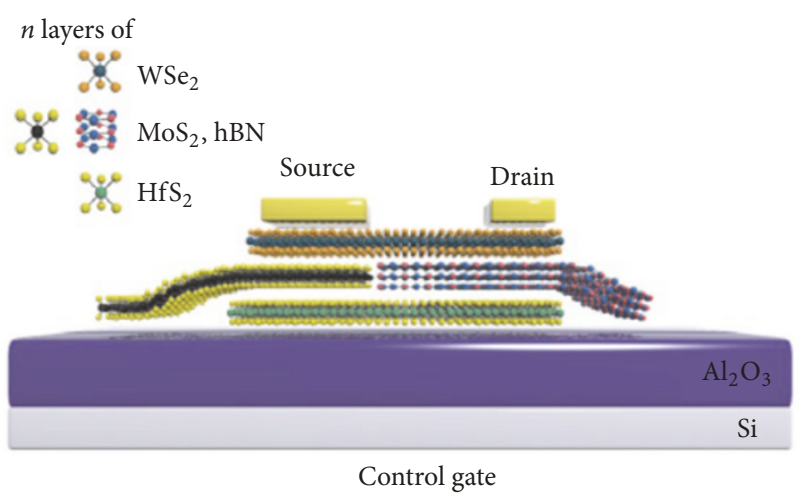

(e)

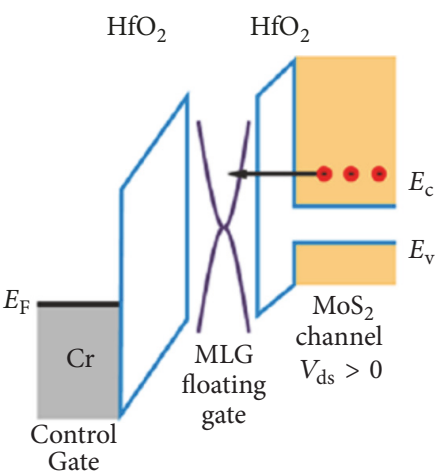

Program operation $\left(V_{\mathrm{cg}}>0\right)$

(b)

Programing

Gr BNS Gr BN MoS 2 Gr BN D
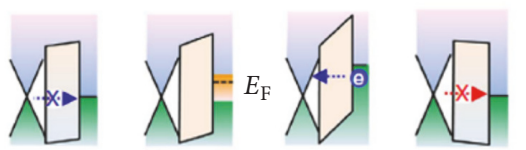

Erasing
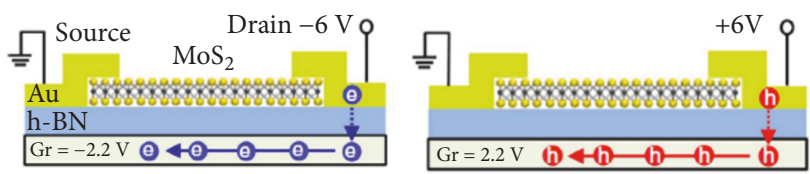

(d)
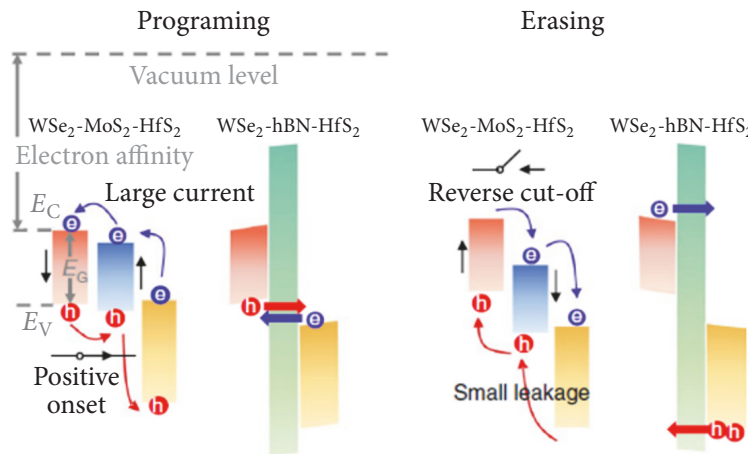

(f)

Figure 2: Floating gate memory based on 2D materials. (a) Schematics and (b) band alignments of programming and erasing processes of a floating-gate memory based on $\mathrm{MoS}_{2}$ /graphene heterostructure. (c) Schematics and (d) Band alignments of programming and erasing processes of a two-terminal floating gate memory based on $\mathrm{MoS}_{2} / \mathrm{h}-\mathrm{BN} /$ graphene heterostructure. (e) Schematics and (f) band alignments of programing and erasing processes of a semifloating gate memory. Reproduced with permissions: (a) and (b) from [36]; (c) and (d) from [37]; (e) and (f) from [28].

for artificial vision, optical demodulators and logic gates for optoelectronic circuits, and synaptic devices for neuromorphic systems. The constructions of ORAMs can be mainly divided into two types according to the device structure: ORAM based on 2D materials and ORAM based on 2D heterostructures, which will be discussed in the Sections 3.2 and 3.3, respectively.
3.1. Nonvolatile ORAM versus Photodetectors. Both ORAMs and traditional photodetectors convert the optical signal to an electronic one. However, the design of ORAMs is in sharp contrast to the photodetectors, in which the photo-generated charge carriers recombine immediately in the photodetectors after the removal of light stimuli, corresponding to an immediate current drop from $I_{p}$ (photo current) to $I_{D}$ (dark 


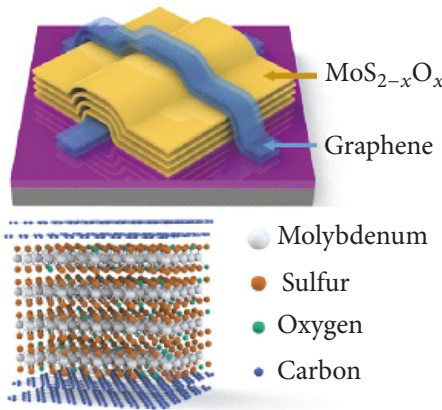

(a)

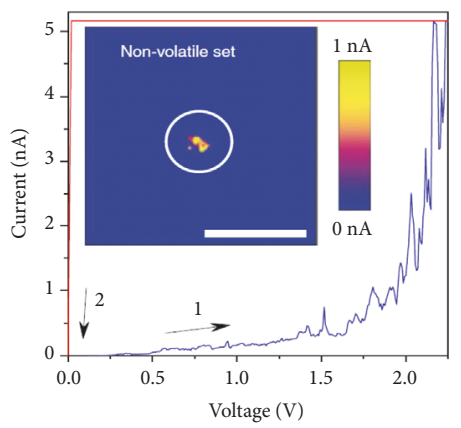

(d)
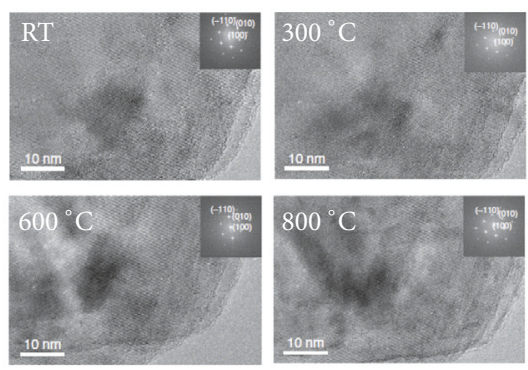

(b)
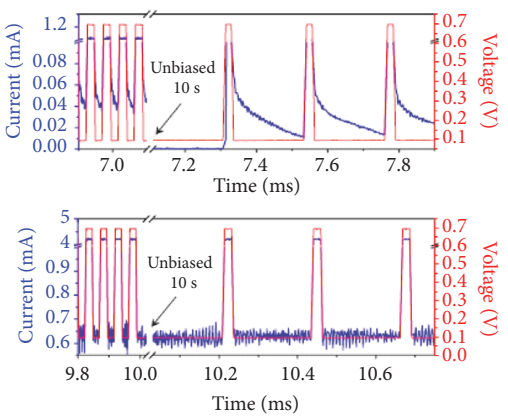

(e)

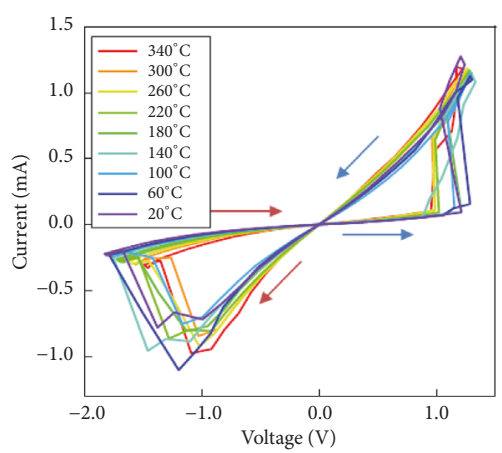

(c)

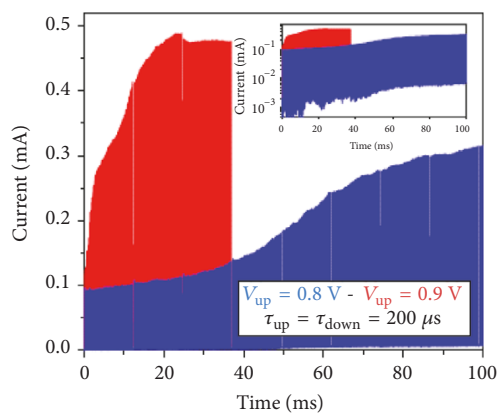

(f)

Figure 3: Two-terminal RRAM devices based on 2D materials. (a) Schematic of the graphene/ $\mathrm{MoS}_{2-\mathrm{x}} \mathrm{O}_{\mathrm{x}} /$ graphene device. (b) HRTEM images of $\mathrm{MoS}_{2-\mathrm{x}} \mathrm{O}_{\mathrm{x}}$ at different temperatures. $\mathrm{MoS}_{2-\mathrm{x}} \mathrm{O}_{\mathrm{x}}$ still maintains a well-defined crystal structure at $800^{\circ} \mathrm{C}$. (c) I-V switching curves of the graphene/ $\mathrm{MoS}_{2-\mathrm{x}} \mathrm{O}_{\mathrm{x}}$ /graphene device at different temperatures. (d) C-AFM test that reveals the formation of conductive filament in $\mathrm{Au} / \mathrm{Ti} / \mathrm{h}-\mathrm{BN} / \mathrm{Cu}$ synapse. (e) and (f) Dynamic responses of Metal/ h-BN/ metal synapses with different programming methods. Reproduced with permissions: (a), (b), and (c) from [29]; (d), (e), and (f) from [39].

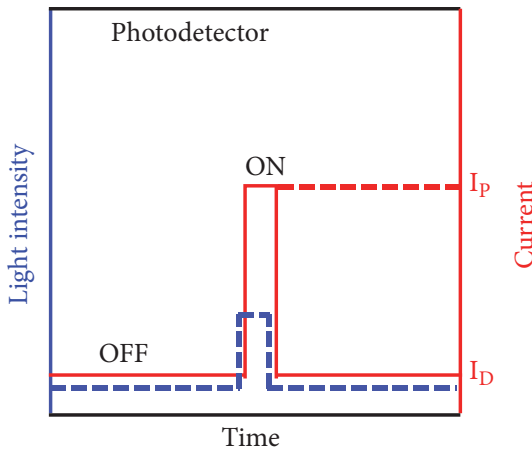

(a)

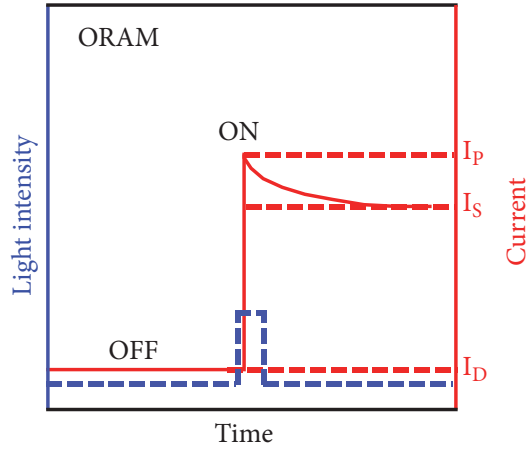

(b)

FIgURE 4: Comparison of photo-responses of (a) conventional photodetectors and (b) ORAMs.

current) (Figure 4(a)). In ORAM, the optical information can be stored and retained for a long period even after the removal of light stimuli, corresponding to a stored and retained photocurrent $I_{S}$ (Figure 4(b)). The responsivity $\mathrm{R}$ in photodetector, which represents the capability of optical-to-electrical conversion, can be expressed according to

$$
\mathrm{R}(\mathrm{A} / \mathrm{W})=\frac{I_{P}-I_{D}}{P \times S}
$$

where $P$ is the light power density and $S$ is the device area. As the photocurrent $I_{p}$ usually suffers from a slight degradation (after the removal of light stimuli) until reaching a stable current storage current of $I_{S}$, here we can define a specific nonvolatile responsivity (NR) for ORAM in a similar way, which can be expressed as

$$
\mathrm{NR}(\mathrm{A} / \mathrm{W})=\frac{I_{S}-I_{D}}{P \times S}
$$

where $I_{S}$ is the storage current. 
3.2. 2D Materials Based ORAM. The working mechanism of ORAMs based on 2D materials mainly lies in providing charge trapping centers for photo-generated charge carriers (electrons/holes), which store the photo-generated electrons/holes even after the removal of light stimuli. In this case, the optical information can be stored in the ORAM. Because the light intensity determines the amount of photogenerated charge carriers, which is generally related to the possibility of charge trapping or interfacial physics. Thus, tuning the light intensity provides an approach to achieve multiple resistance states. Additionally, light stimuli with different wavelengths correspond to different optical excitation energies and different amount of light absorption, which may also induce to different charge trapping/detrapping physics and multiple resistance states.

In 2014, Lei et al. demonstrated multilayered $\mathrm{CuIn}_{7} \mathrm{Se}_{11}$ based ORAM array for image sensing, and further extended to other atomically thin 2D materials (InSe and $\mathrm{MoS}_{2}$ ) [40]. The design concept of the ORAM is to build potential well with an energy band bending between 2D material channel and metal electrodes, trapping photo-generated electrons. Although the ORAM exhibits good photo-response, its short retention time (less than $50 \mathrm{~s}$ ) and low ON/OFF ratio (less than 10) limit the applications for multilevel nonvolatile memories. Further, Lee et al. introduced artificially charge traps at the $\mathrm{MoS}_{2} / \mathrm{SiO}_{2}$ interface through $\mathrm{O}_{2}$ plasma treatment of $\mathrm{SiO}_{2}$ before $\mathrm{MoS}_{2}$ monolayer growth (Figure 5(a)). The band alignments of the initial state, upon light exposure and reading process for $\mathrm{ON}$ and $\mathrm{OFF}$ states are illustrated in Figure 5(b). At the initial state, a potential well is formed by metal-semiconductor Schottky barrier with the applied gate voltage. A positive gate voltage pulse first programs the device to OFF state by driving the electrons in the channel to the interfacial trap states, leading to a low OFF current of $4 \mathrm{pA}\left(\mathrm{V}_{\mathrm{sd}}=3 \mathrm{~V}\right)$. Upon light illumination (450 $\mathrm{nm}$ laser), photo-generated electrons are accumulated in the potential well, while holes escape from both electrodes through upward energy band bending. After the removal of light illumination, the readout process (readout bias is applied) eliminates one of the Schottky barriers and releases the stored electrons. By intentionally introducing charge traps, the $\mathrm{MoS}_{2}$ based ORAM device exhibits improved ON/OFF ratio of 4700 and retention time of $6000 \mathrm{~s}$. However, the photo-current still suffers from relatively large degradation after the removal of light exposure [41].

Towards the design of ORAMs with high photoresponsivity, large ON/OFF ratio, low dark current and good retention properties, researchers adopted other charge trapping methods to fabricate multilevel and highly responsive nonvolatile 2D ORAM. Cho group successfully reported a multibit $\mathrm{MoS}_{2}$ ORAM with high ON/OFF ratio of $10^{7}$, long retention time over $10^{4} \mathrm{~s}$, and high nonvolatile responsivity exceeding $8000 \mathrm{~A} / \mathrm{W}$ at an illumination power of $0.1 \mu \mathrm{W}$ [42]. They employed a layer of $\mathrm{Au}$ nanoparticles as the charge trapping layer and floating gate between the blocking and tunneling dielectrics to store photo-generated charge carriers in the $\mathrm{MoS}_{2}$ ORAM (Figure 5(a)). The $\mathrm{MoS}_{2}$ ORAM can be programmed in both electrical and optical manner, which enables versatile applications, such as performing logic operations and coincidence detection [3]. The schematic band diagrams of electrical and optical programming are illustrated in Figures 5(d) and 5(e), respectively. For the electrical programming, a negative gate voltage induces the transfer of electrons in Au nanoparticle layer to the conduction band of $\mathrm{MoS}_{2}$ layer through Fowler-Nordheim tunneling; for the optical programming, the electrons can be easily transferred from Au nanoparticle layer to $\mathrm{MoS}_{2}$ valence band with the light illumination (655 nm laser), which prevents the recombination of photo-generated electrons and holes in the $\mathrm{MoS}_{2}$ layer. The programmed resistance states can be erased by applying a positive gate voltage. The $\mathrm{MoS}_{2}$ ORAM exhibits light intensity-dependent multilevel resistance states, integrating both optical sensing and nonvolatile data storage (Figure 5(f)).

In addition to the typical FET ORAMs, ferroelectric ORAM based on monolayer $\mathrm{MoS}_{2}$ has also exhibited unique characteristics, including nondestructive data readout and low operating voltage [43] (Figures 6(a) and 6(b)). The optical illumination can induce the domain re-arrangement in the $\mathrm{MoS}_{2}$-PZT ORAM, which further causes the change in conductivity. The ferroelectric ORAM can be globally optically SET and electrically RESET or electrically SET and optically RESET, depending on the initial polarization states of PZT (Figure 6(c)). Wang et al. fabricated a two-terminal $\mathrm{MoS}_{2}$ ORAM with a structure of $\mathrm{Au} / \mathrm{MoS}_{2}$ nanospheres/Au [44]. With a poling voltage of $6 \mathrm{~V}$, upon the light illumination the device can be switched from HRS to LRS at $-2.9 \mathrm{~V}$ with an $\mathrm{ON} / \mathrm{OFF}$ ratio of 10 . A positive voltage of $4.2 \mathrm{~V}$ can switch the device back to HRS. By combining different poling voltage and light illumination, multilevel resistance states can be obtained in the two-terminal $\mathrm{MoS}_{2}$ ORAM device, which provides possibilities to the design of two-terminal ORAM based on 2D materials.

\subsection{D Heterostructure Based Nonvolatile ORAM. The $2 \mathrm{D}$} material based ORAMs are generally sensitive to environmental factors because most of the memory functions in ORAMs are dependent on the charge trapping carriers in original or artificial defects at the materials interface. The charge trapping mechanism usually leads to relatively short retention time in the memory device. By combining the $2 \mathrm{D}$ materials with other semiconductors, the ORAMs based on 2D heterostructure can widen ORAM design possibilities and improve the ORAM performance, including the light absorption range, environmental stability and responsivity, nonvolatile characteristics and data storage capability.

Roy et al. reported an ORAM based on graphene/ $\mathrm{MoS}_{2}$ vertical heterostructure, which demonstrates a high photoresponsivity of $5 \times 10^{8} \mathrm{~A} / \mathrm{W}$ at room temperature. However, the switching ON/OFF ratio is less than 2, owing to the high electrical conductivity of graphene and the large dark current of the device. The low ON/OFF ratio restricts the application for multibit ORAM [45]. Later, Du et al. designed a broadband (ultraviolet to visible region) ORAM based on $\mathrm{WSe}_{2} / \mathrm{h}-\mathrm{BN}$ heterostructure (Figure $7(\mathrm{a})$ ), which exhibits a large $\mathrm{ON} / \mathrm{OFF}$ ratio of $1.1 \times 10^{6}$, over 128 distinct resistance states and remarkable retention time over $4.5 \times 10^{4} \mathrm{~s}$ [46]. 


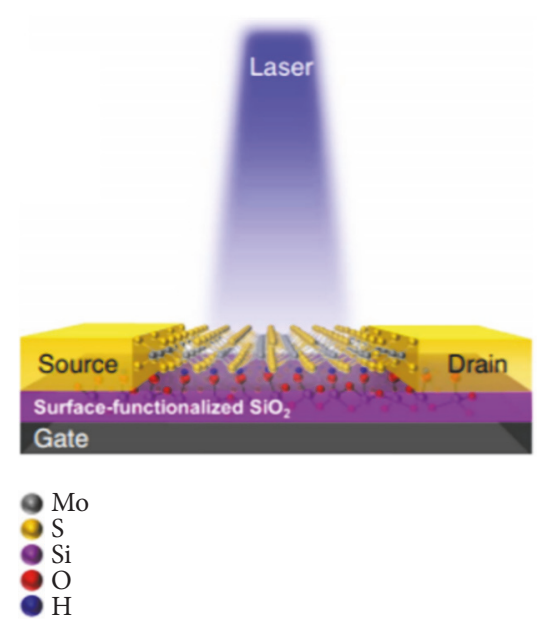

(a)

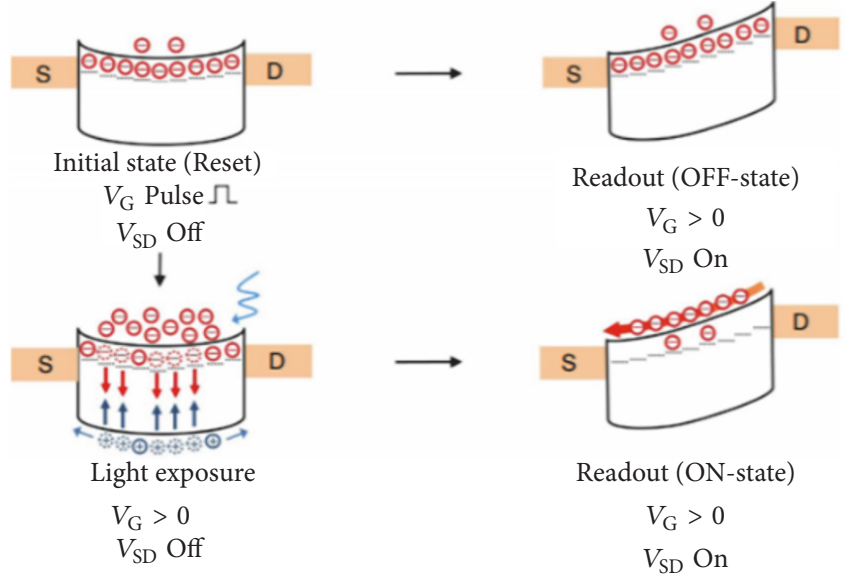

(b)
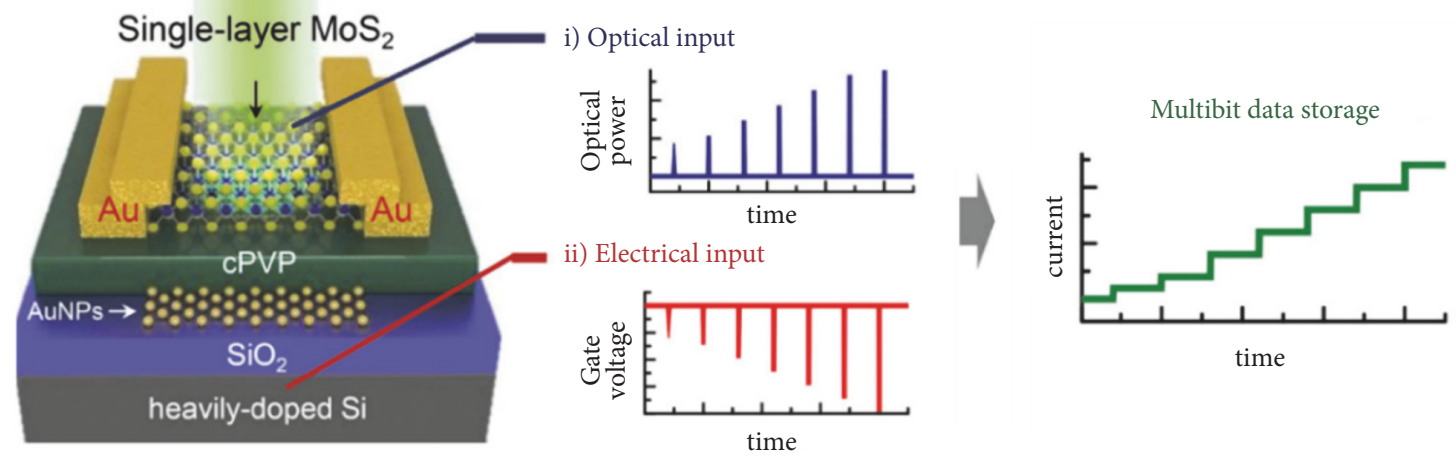

(c)

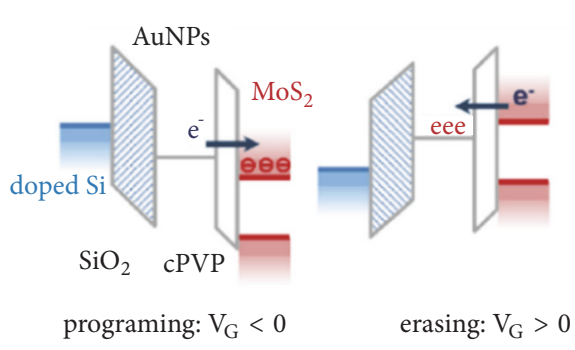

(d)

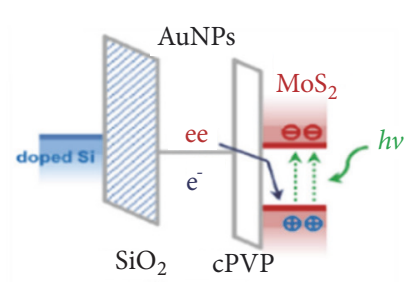

programing: $\mathrm{V}_{\mathrm{G}}<0$

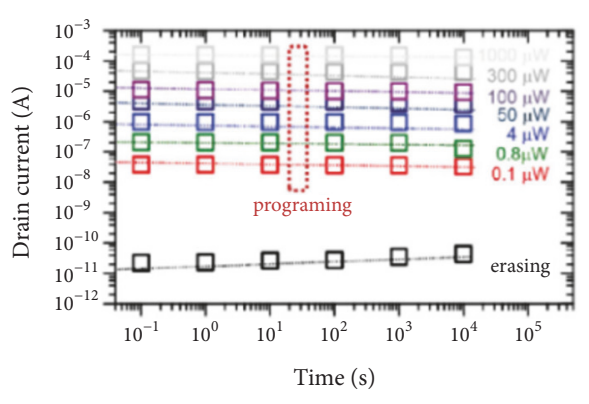

(f)

FIGURE 5: (a) Schematic illustration of $\mathrm{MoS}_{2}$ ORAM with functionalized $\mathrm{MoS}_{2} / \mathrm{SiO}_{2}$ interface. (b) Band diagrams of the initial state, upon light exposure and reading process. (c) Schematic illustration of $\mathrm{MoS}_{2}$ ORAM with Au nanoparticles as charge trapping layer. (d) Band diagram of electrically-controlled programming and erasing. (e) Band diagram of light-controlled programming. (f) Light-controlled multilevel resistance states. Reprodued with permissions: (a) and (b) from [41]; (c), (d), (e), and (f) from [42].

Upon the light illumination (405 $\mathrm{nm}$ laser), the photogenerated electrons in $\mathrm{BN}$ conduction band can be transferred to $\mathrm{WSe}_{2}$ conduction band under a negative gate voltage of $-20 \mathrm{~V}$ (programming process). The photo-generated holes are left and localized in the middle of BN bandgap even after the removal of light stimulation and electric field, which indicates the nonvolatile switching characteristics. A positive gate voltage with light illumination erases the device and turns the device back to the initial states by driving holes from $\mathrm{BN}$ to $\mathrm{WSe}_{2}$ (Figure 7(b)). Figure 7(c) illustrates 130 resistance states obtained by applying an increased number of light pulses. The $\mathrm{WSe}_{2} / \mathrm{h}-\mathrm{BN}$ can respond to the lights with a wide range of wavelength from $410 \mathrm{~nm}$ to 750 $\mathrm{nm}$. A color image sensor array integrated with sensing 


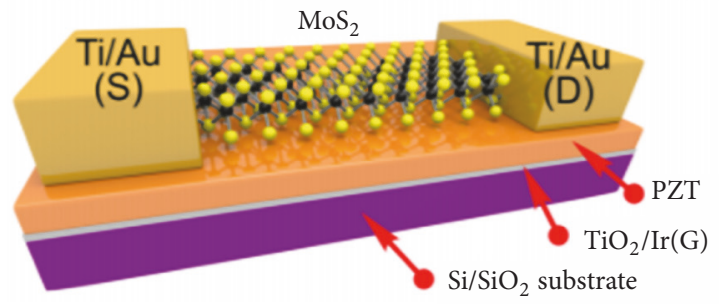

(a)

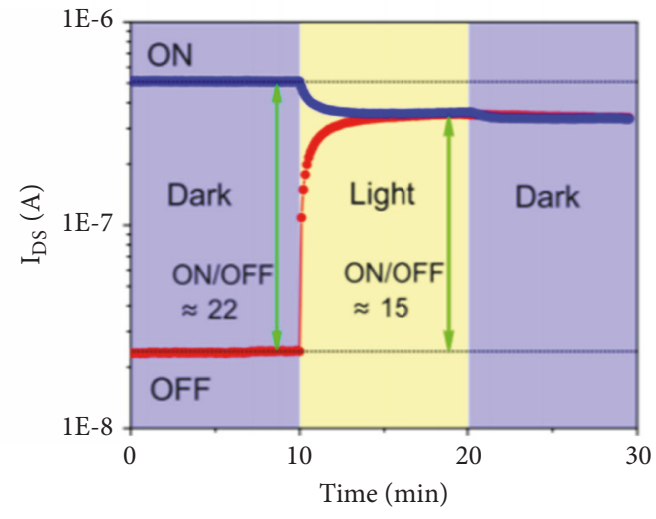

(b)

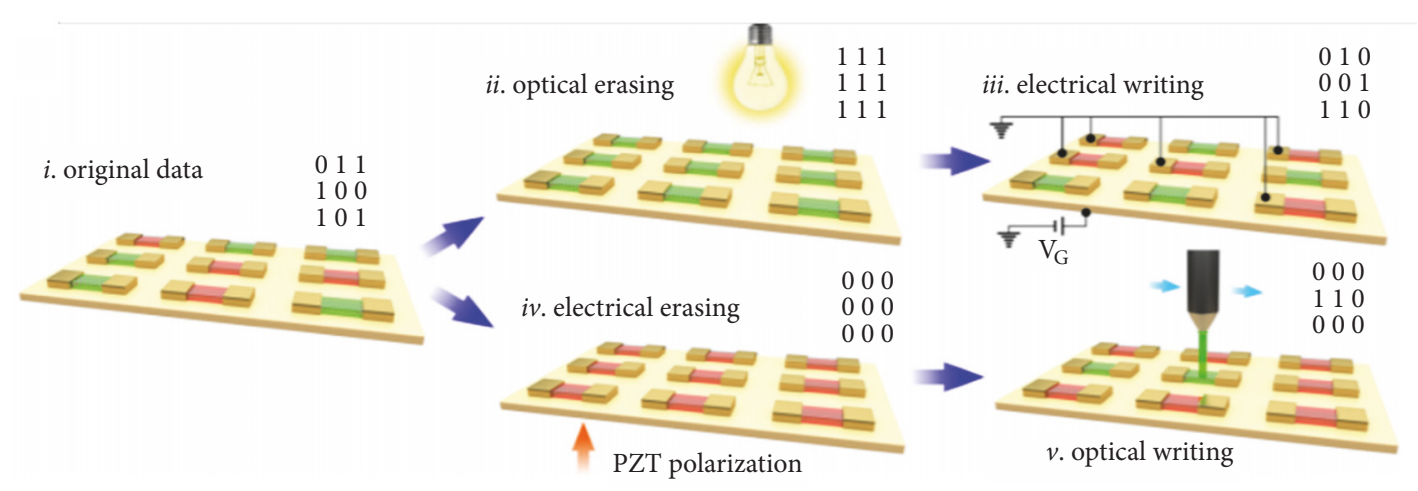

(c)

Figure 6: (a) Schematic of $\mathrm{MoS}_{2}$ ferroelectric ORAM. (b) Retention characteristics after the removal of light stimuli. (c) Global operations of "optical erase and electrical write" and "electrical erase and optical write." Reproduced with permission from [43].

and storage capability exhibits promising potentials for the future design of smart image sensor (Figure 7 (c) inset). The heterostructure configuration can also be extended to other materials, such as BP/ BN based ORAM, which exhibits ultrahigh nonvolatile responsivity of $1.2 \times 10^{7} \mathrm{~A} / \mathrm{W}$ [46]. However, the three-terminal FET ORAM usually involves with high programming gate voltage, relatively low integration level and complex circuitry for integration. Two-terminal ORAM is highly desirable for the design of the device with simple structure, high integration level and low power consumption.

To further decrease the programming voltage and power consumption in ORAM device, Tran et al. demonstrated a nonvolatile multibit ORAM based on $\mathrm{MoS}_{2} / \mathrm{BN} /$ graphene van der Waals heterostructure, showing a programming voltage (source-drain voltage) of $-10 \mathrm{~V}$, extremely low darkcurrent of $10^{-14} \mathrm{~A}$ and high nonvolatile responsivity of $2 \times 10^{4}$ A/W (@458 nm, 2nW) (Figure 7(c)) [47]. A negative sourcedrain voltage of $-10 \mathrm{~V}$ induces the electrons tunneling through $\mathrm{BN}$ to graphene floating gate. The accumulated electrons in the graphene layer serve as a negative gate bias, thus programs the device with extremely low dark current (LRS). The visible light pulses then induce the photo-generated holes in $\mathrm{MoS}_{2}$ layer, tunneling through the low hole barrier of $\mathrm{MoS}_{2} / \mathrm{BN}$ and combining with the stored electrons in the graphene layer, and RESET the device to HRS (Figure 7(d)). This operation scheme leads to a high ON/OFF ratio of $10^{6}$ and over 16 distinct storage levels by tuning the light intensities [47]. Hu et al. reported a two-terminal and vertical ORAM based on $\mathrm{BP} / \mathrm{ZnO}$ nanoparticles heterojunction, exhibiting broadband absorption from ultraviolet $(380 \mathrm{~nm})$ to near infrared $(785 \mathrm{~nm})$ and low SET voltage $<4 \mathrm{~V}$ (Figure $7(\mathrm{e})$ ). The optically-controlled resistive switching originates from fast electron/hole separation in the heterojunction, which is further involved in the manipulation of the formation/rupture of oxygen vacancy filament [48].

Most of the absorption spectrum in ORAM device currently is limited in the ultraviolet and visible region. As infrared radiation is the communication medium for data transfer in wireless networks, night vision, image sensing, medical diagnosis, etc., the extension of absorption to the infrared region in ORAM is highly desirable, which not only enables the conversion and storage of infrared data, but also benefits for the optical data communication in the photonic circuit and artificial visual intelligence. The application of $2 \mathrm{D}$ heterostructures provides possibilities in designing infrared ORAMs by combining $2 \mathrm{D}$ materials with small bandgap 


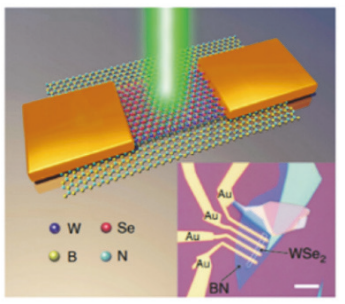

(a)

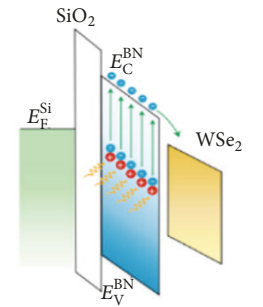

Program $(\mathrm{Vg}<0$, light $)$

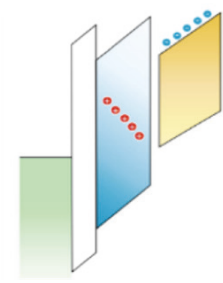

$\operatorname{Read}(\mathrm{Vg}>0$, dark)

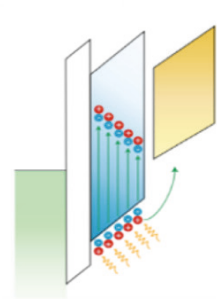

Erase $(\mathrm{Vg}>0$, light $)$

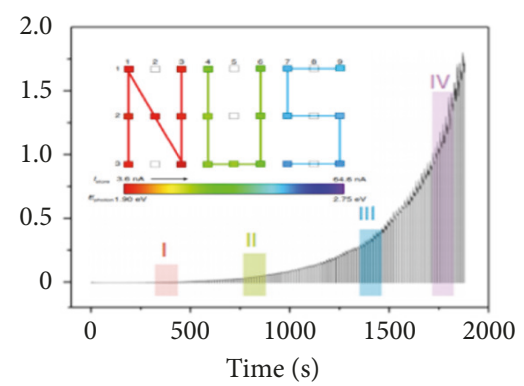

(c)

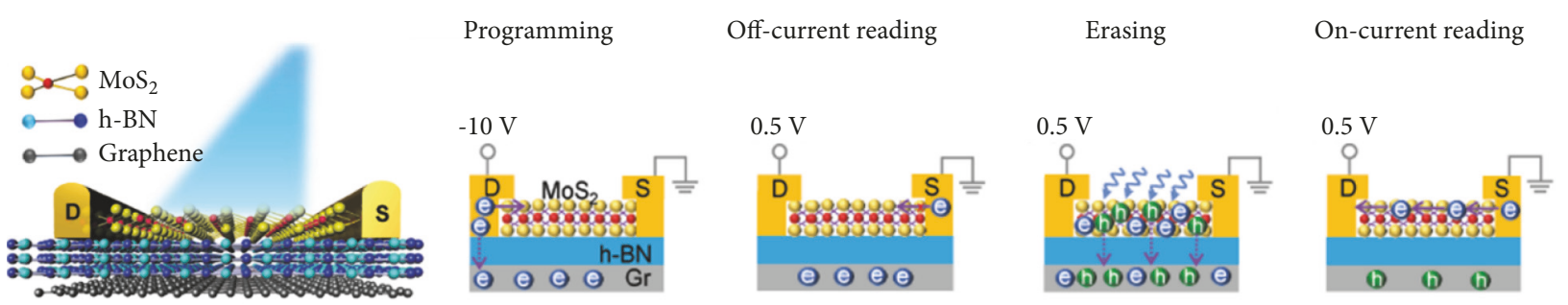

(d)

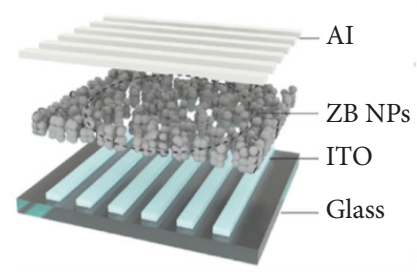

(e)

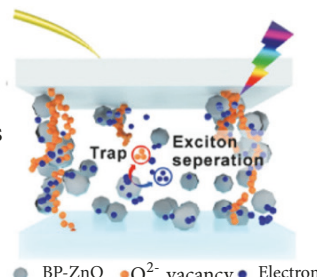

$\mathrm{BP}-\mathrm{ZnO} \bullet \mathrm{O}^{2-}$ vacancy $\bullet$ Electron

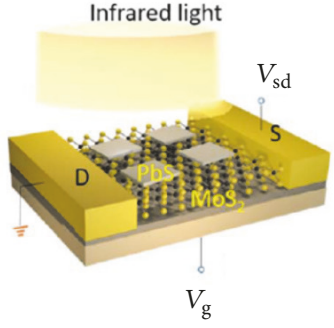

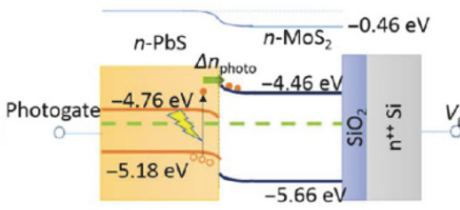

(f)

FIGURE 7: (a) Schematic structure and (b) schematic band diagrams of switching mechanism in WSe $2 /$ h-BN ORAM. (c) 130 storage states obtained by applying a different number of $405 \mathrm{~nm}$ light pulse (duration of $0.5 \mathrm{~s}$ and intensity of $210 \mathrm{~mW} / \mathrm{cm}^{2}$ ). The gate voltage is - $80 \mathrm{~V}$. Inset: integrated $\mathrm{WSe}_{2} / \mathrm{h}$-BN pixel matrix for color image sensor with memory capability. Two-terminal ORAMs based on different heterostructures. (d) Schematics of ORAM with $\mathrm{MoS}_{2} / \mathrm{h}-\mathrm{BN} /$ graphene heterostructures and illustration of programming, erasing and ON/OFF readout processes. (e) Schematics of ORAM with phosphorene/ZnO nanoparticles heterostructure and illustrations of the SET process with external bias and light illumination. (f) Schematic of $\mathrm{MoS}_{2} / \mathrm{PbS}$ infrared ORAM and band diagram of $\mathrm{MoS}_{2} / \mathrm{PbS}$ heterostructure upon the infrared laser illumination. Reprodued with permissions: (a), (b), and (c) from [46]; (d) from [47]; (e) from [48]; (f) from [49].

semiconductors or up-conversion nanoparticles. Wang et al. reported a nonvolatile infrared ORAM by adopting a $\mathrm{MoS}_{2} / \mathrm{PbS}$ van der Waals heterostructure, which can respond to a wide wavelength range from $880 \mathrm{~nm}$ to $1940 \mathrm{~nm}$ (Figure 7(f)) [49]. The optical switching originates from the localized photo-generated holes in $\mathrm{PbS}$ near the interface and the injection of photo-generated electrons from $\mathrm{PbS}$ to $\mathrm{MoS}_{2}$, thus modulating the $\mathrm{MoS}_{2}$ conductivity. A retention time of over $10^{4} \mathrm{~s}$ and multi-level storage states can be obtained by applying $1940 \mathrm{~nm}$ infrared light pulses. The nonvolatile responsivity reaches up to $1.2 \times 10^{4} \mathrm{~A} / \mathrm{W}$. The upconversion nanoparticles based on lanthanide ions-doped $\mathrm{NaYF}_{4}$ with narrow absorption band at $980 \mathrm{~nm}$ also provide possibilities for $2 \mathrm{D}$ materials based infrared ORAM. The up-conversion nanoparticles/ $\mathrm{MoS}_{2}$ serve as charge trapping and separation centers upon the infrared light illumination, which is responsible for the optical assisted switching process [50]. The up-conversion nanoparticles/ $\mathrm{MoS}_{2}$ based infrared ORAM enables to sense and store the $980 \mathrm{~nm}$ infrared light with different light intensities, corresponding to different resistance states.

Table 1 compares the optical switching characteristics of nonvolatile ORAMs based on 2D materials and their heterostructures with other material systems. The nonvolatile ORAMs based on 2D materials have exhibited high responsivity, low optical power consumption, large ON/OFF ratio, good endurance, and broadband absorption compared with that based on other materials (e.g., oxides, hybrid 


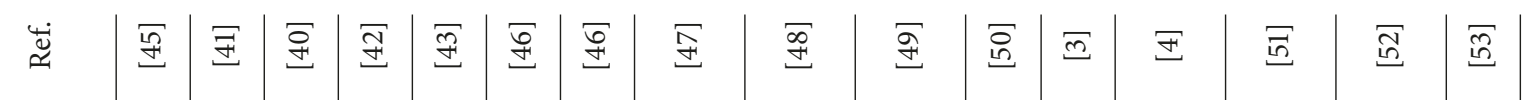

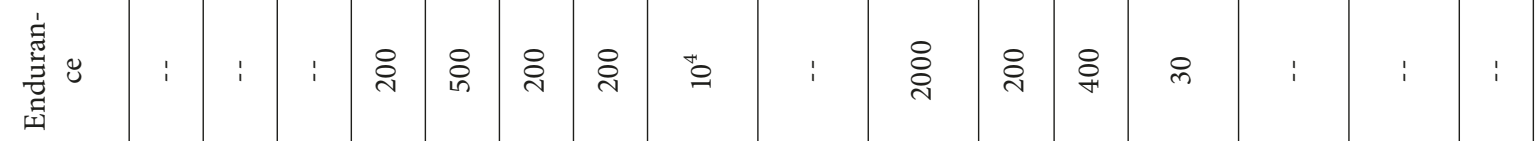

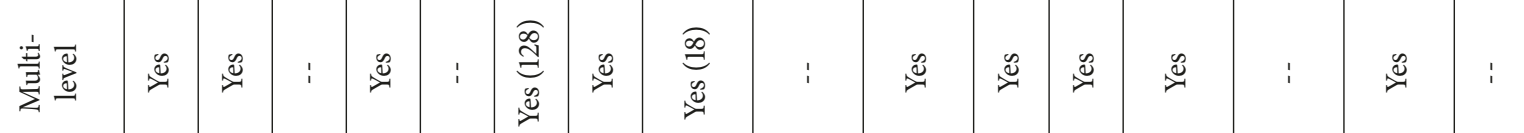

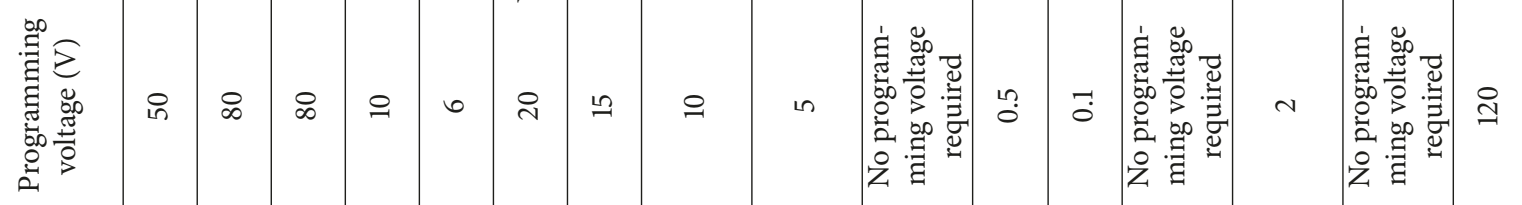

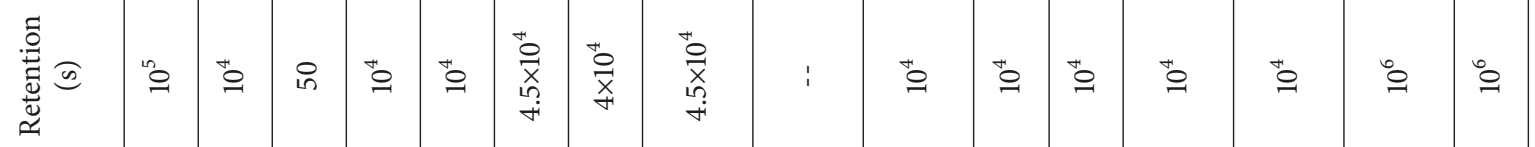

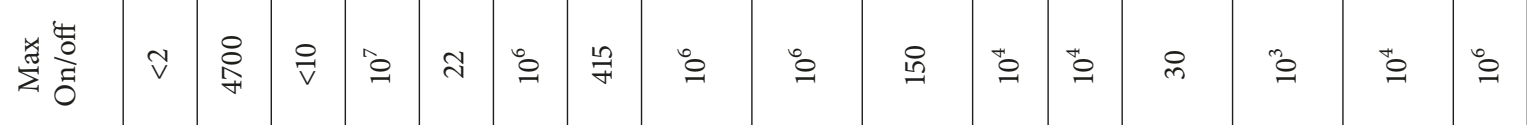

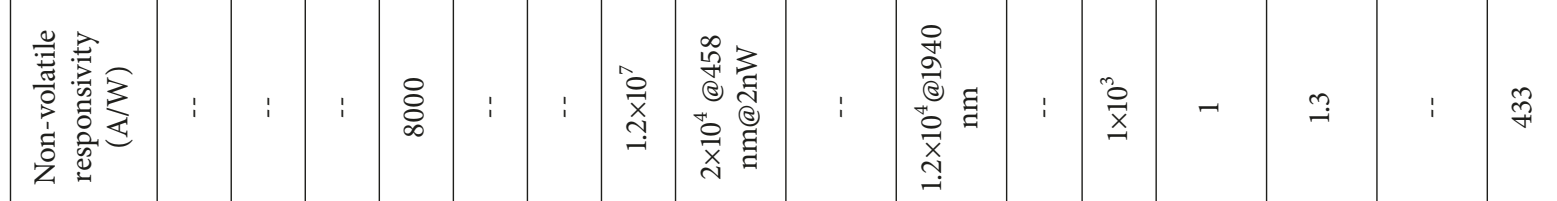

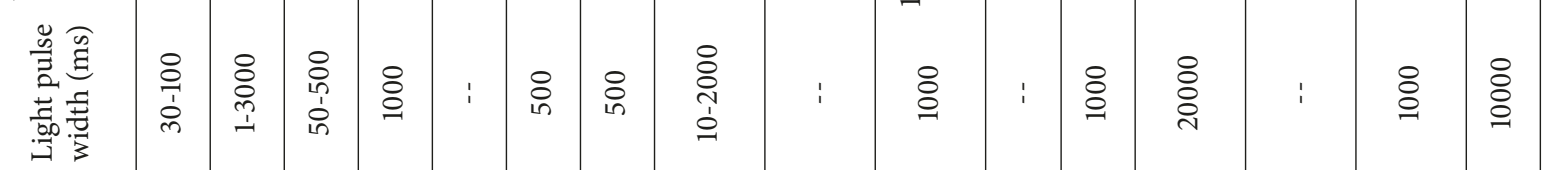

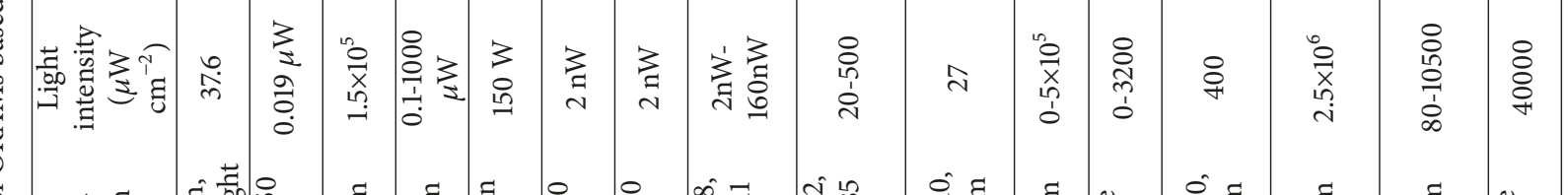

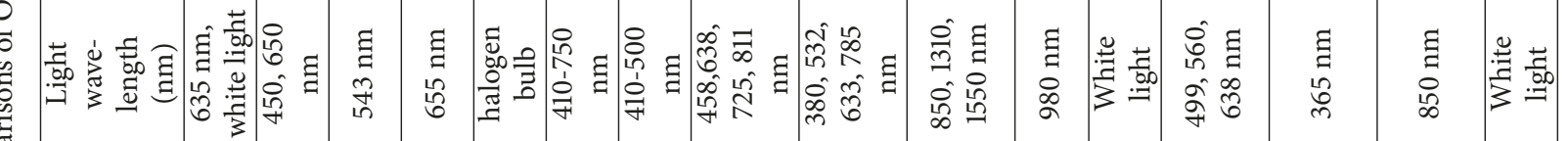

$$
\begin{aligned}
& \text { 空 }
\end{aligned}
$$

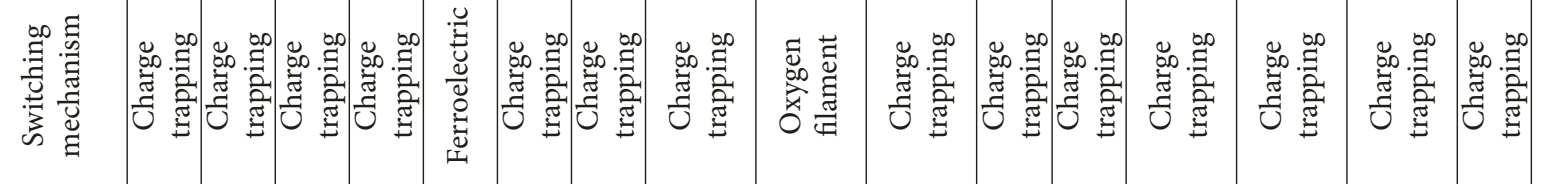

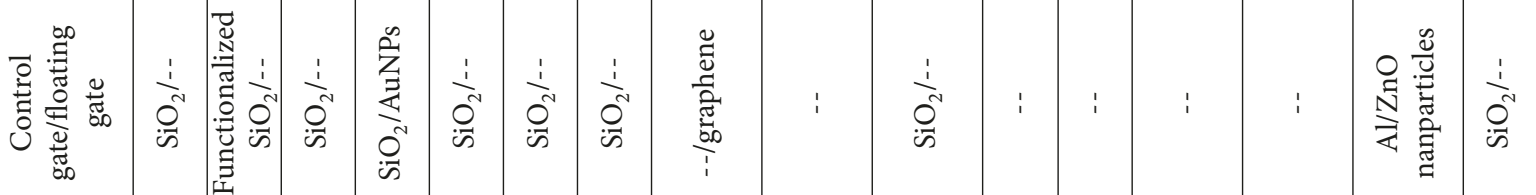

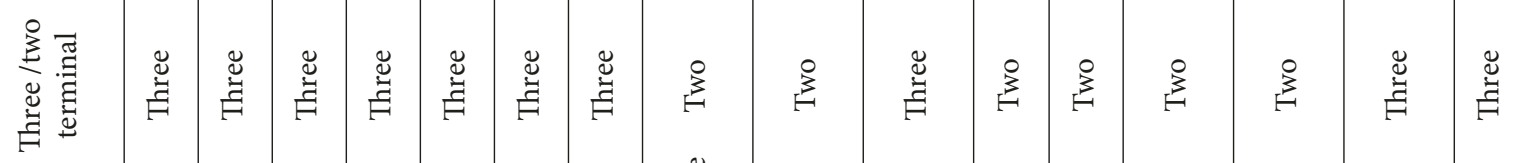

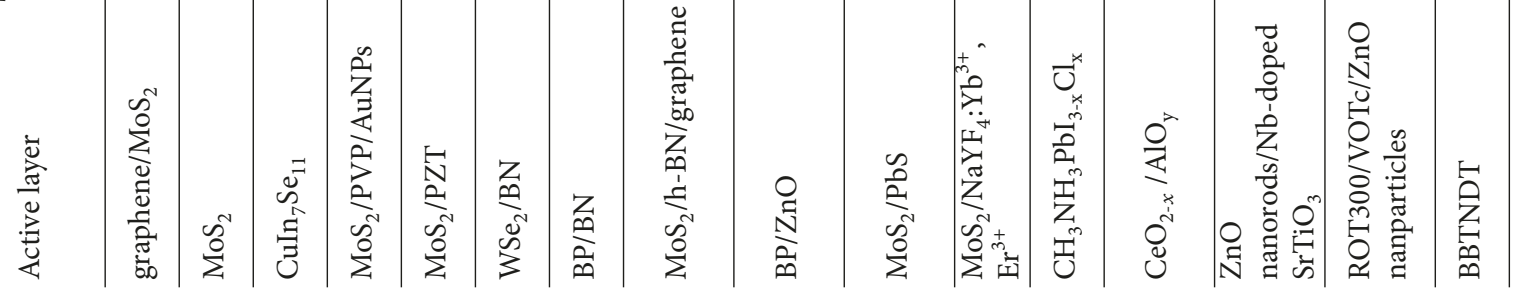




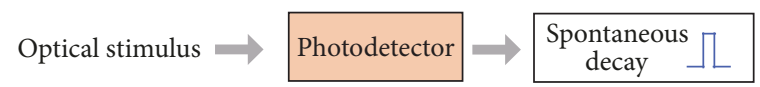

(a)

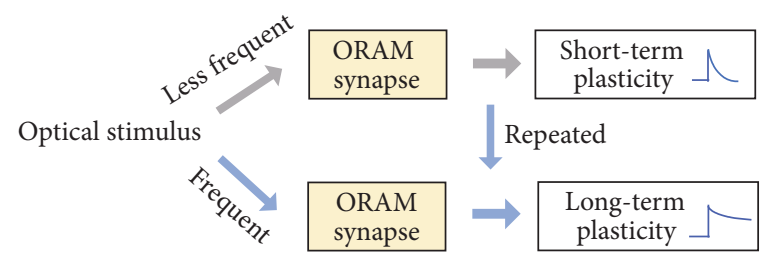

(b)

FIGURE 8: Comparison of photo-responses of (a) photodetectors without tunable plasticity and (b) ORAM synaptic device with light-tunable plasticity.

organic-inorganic perovskite, organic materials), however, the high programming voltage in most of $2 \mathrm{D}$ material ORAM is required to be further lowered to achieve a low power consumption in the future.

\section{2D Materials Based ORAM Synaptic Device}

4.1. ORAM Synaptic Device versus Photodetector. The integration of optical sensing and data storage in ORAM enables direct storage of optical information from the outside world without optical-to-electrical conversion, however, these functions are restricted in the digital applications. Towards the future low power neuromorphic computing, ORAM synaptic devices are desirable that provide possibilities to bridge the sensing and neuromorphic computing and the direct processing of optical information (e.g., image processing) [18]. In comparison to conventional photodetectors and nonvolatile ORAMs, ORAM synaptic devices exhibit both opticallycontrolled nonvolatile and volatile switching characteristics. It can respond to optical stimuli and exhibit light-tunable synaptic behaviors, such as STP, LTP and STDP. Figure 8 illustrates the light modulating schemes in photodetectors (a) and ORAM synaptic devices (b). In sharp contrast to the conventional photodetector or image sensors, ORAM synaptic devices sense information in ways like neural signals with light-tunable and time-dependent plasticity, implicating the potential application in the construction of neuromorphic visual systems.

4.2. ORRAM Synaptic Device. Qin et al. first reported an ORAM synaptic device based on graphene/carbon nanotube (CNT) heterostructure, demonstrating light-tunable synaptic behaviors, such as, light-tunable STP, LTP, STLR and STDP (Figure 9(a)) [54]. The channel conductance can be tuned through optical pulse and gate pulse. The multiple state conductance can be regarded as the biological synaptic weight. Figure 9(b) shows the STP stimulated with an optical pulse (405 nm, $50 \mu \mathrm{W}, 5 \mathrm{~ms}$ ), where the conductance increases abruptly and relaxes within seconds. The STP is triggered owing to the electron transfer from CNT to graphene upon the light illumination, tuning the built-in field formed at graphene/CNT interface. Optically tuned paired-pulse facilitation (PPF) function is an important function for temporal visual information encoding, as illustrated in Figure 9(c). The plasticity can be further modulated to LTP by combining a gate voltage and an optical pulse (Figure $9(\mathrm{~d})$ ). The LTP originates from the trapping of photo-generated holes from graphene or SWCNT at the $\mathrm{SiO}_{2}$ surface owing to the defects. A STDP learning rule (symmetric STDP) is also demonstrated in this work (Figure 9(f)), which is related to associative learning and enables to the application of coincidence detector for spiking activity. Both the nonHebbian learning rule (e.g., STLR) and Hebbian learning rule (e.g., STDP) are mimicked (Figures 9(e) and 7(f)), respectively. The implementations of STLR and STDP are associated with the spatiotemporal information processing in the neural network. Similar STLR and STDP learning rules can also be performed in ion-gated 2D ORAMs $[55,56]$.

Other types of 2D materials heterostructures have also been utilized for ORAM synaptic devices, such as $\mathrm{MoS}_{2} /$ perylene-3,4,9,10-tetracarboxylic dianhydride (PTCDA) heterojunction, exhibiting large synaptic weight change and PPF ratio [22]. To further broaden the absorption spectrum and reduce the power consumption, a hybrid structure of 2D $\mathrm{WSe}_{2}$ /boron-doped Si nanocrystals was employed for ORAM synaptic device with wide absorption spectrum from ultraviolet to near-infrared and low power consumption of $75 \mathrm{fJ}$, which points the way to the development of low-power artificial visual intelligence beyond human eyes [57]. Table 2 compares the basic performances of ORAM synaptic devices based on $2 \mathrm{D}$ materials with other different active layers.

\subsection{Applications in Neural Network and Image Processing.} The multiple synaptic functions demonstrated in the ORAM synaptic device facilitate the coupling of optical sensing and neuromorphic computing, and enable the applications in the optical neural network for image processing (e.g., pattern recognition). Generally, for the conventional image processing based on neuromorphic computing, the optical signals in the images are first converted to electronic signals through image sensors. The electronic signals are then transferred to the synaptic arrays in the neural network for further processing. In contrast to the conventional memory synaptic devices operated by electrical method, the ORAM 


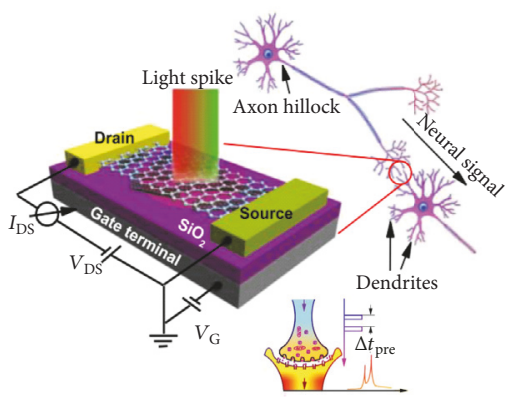

(a)

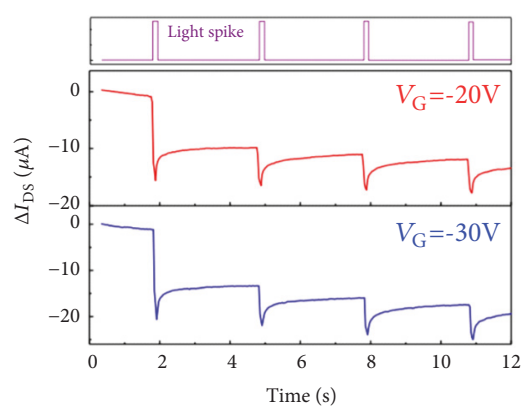

(d)

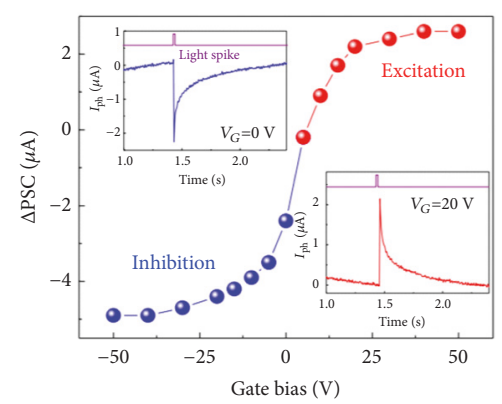

(b)

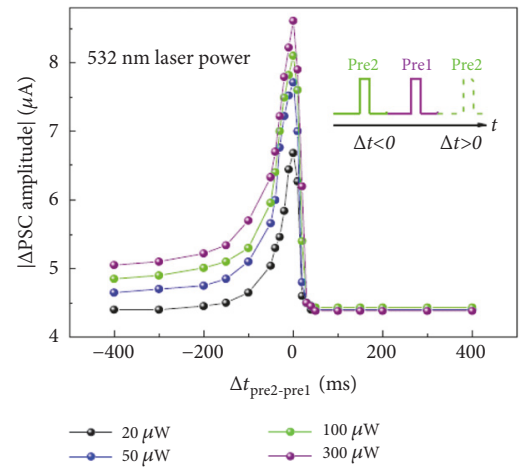

(e)

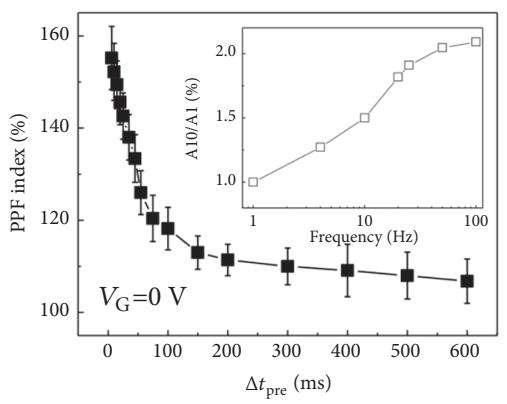

(c)

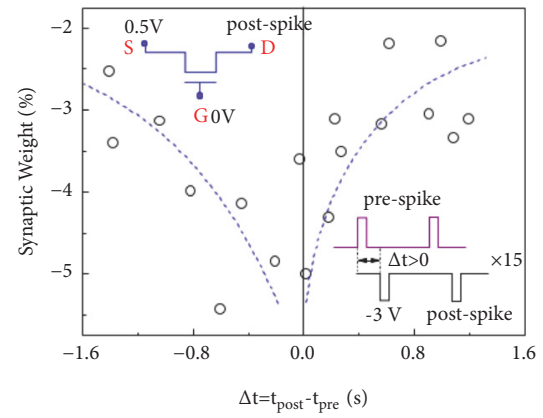

(f)

FIGURE 9: (a) Schematics of graphene/CNT based ORRAM synaptic device. (b) STP: post-synaptic current stimulated with an optical pulse at various gate bias. (c) PPF index as a function of pulse interval. (d) LTP obtained by combining optical pulse and voltage pulse. (e) Illustration of STLR learning rule. (f) Illustration of STDP learning rule. Reproduced with permission from [54].

synaptic devices respond to the optical stimuli directly and exhibit light-evoked synaptic behaviors. The constructed optic-neural network through ORAM synaptic devices can potentially simplify the circuitry for neuromorphic image processing and reduce the power consumption during the data transmission. Additionally, the high integration level in ORAM synaptic device provides future miniaturized and power-efficient artificial eye design [18]. Tian et al. first developed an optic-neural network (ONN) with optical synapses based on graphene/2D perovskite, for which the pattern recognition rate in this ONN can reach up to $80 \%$ [58]. Seo et al. further demonstrated an ONN for mimicking color-mixed pattern recognition in human vision. The ORAM synaptic device is integrated with a $\mathrm{WSe}_{2} / \mathrm{h}-\mathrm{BN}$ photodetector and a synaptic device based on $\mathrm{WSe}_{2} /$ weight control layer (WCL)/h-BN (Figure 10). The optical synapse can respond to the lights with different wavelengths (red, green and blue), showing different synaptic weight change for long-term potentiation/long-term depression (Figure 10(b)). According to the wavelength-dependent synaptic weight change, an ONN (Figure 10(c)) is developed for the task of color-mixed pattern recognition, which differentiates from the conventional neural network (NN) without wavelengthdependent synaptic weight control. The recognition rate for mixed-color digits (Figure $10(\mathrm{~d})$ ) in $\mathrm{ONN}$ is around $60 \%$ higher than that in NN [64].

\section{Conclusions and Perspectives: 2D Materials in Designing Neuromorphic Devices}

Owing to the strong light-matter interaction, unique physical properties, electrostatic tunability and downward scaling potential, 2D materials and their heterostructures have been widely employed for optoelectronics and memory applications in recent years. Towards the trend of functional diversification in "MtM", the integration of optical sensing, data storage and processing drives the design of ORAM and optoelectronic synaptic devices, which can potentially simplify the circuit complexity and reduce the power consumption for the photonic circuit and artificial vision. In contrast to the conventional image sensors with light-intensity sensing, the ORAM device with light tunable plasticity (lightdosage sensing) can further be applied for the construction of ONN for pattern recognition, potentially bridging the sensory data and neural network in the neuromorphic visual system. The nonvolatile ORAMs based on 2D materials heterostructures have exhibited high photoresponsivity, stable multilevel data storage and good retention and endurance properties. Although ORAM devices based on 2D materials have exhibited various advantages over other materials, the high programming voltage is still a main issue, which causes high power consumption. Additionally, the three-terminal structure in most of the $2 \mathrm{D}$ material ORAMs restricts the 


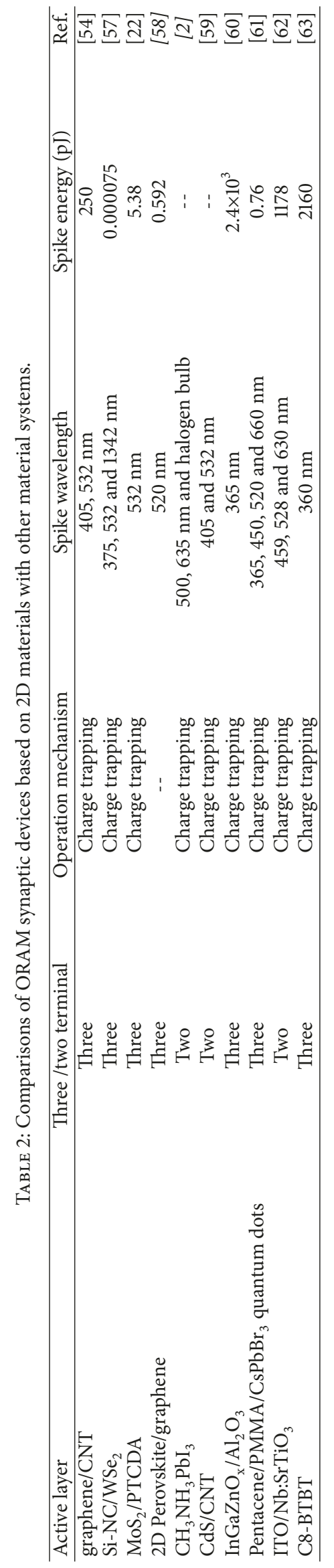




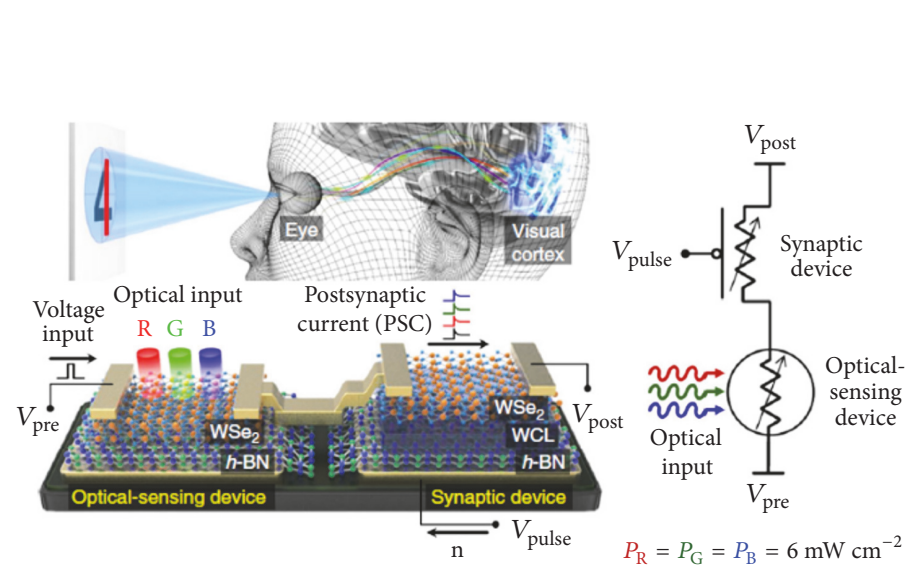

(a)

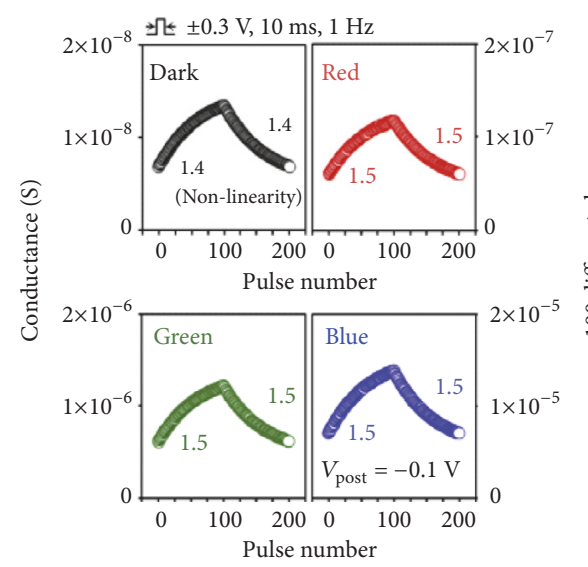

(b)

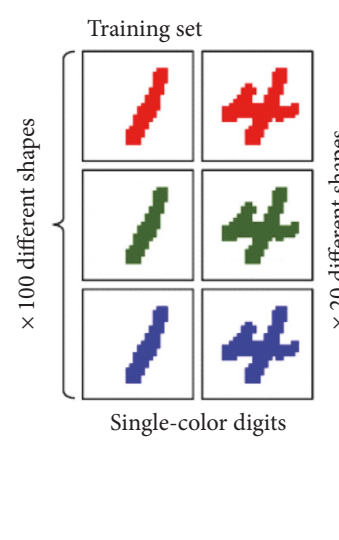

(d)

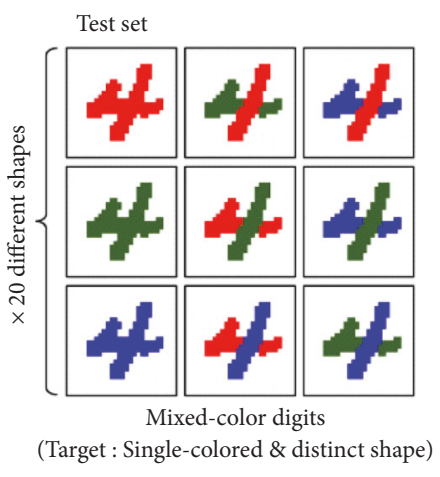

(Target : Single-colored \& distinct shape)

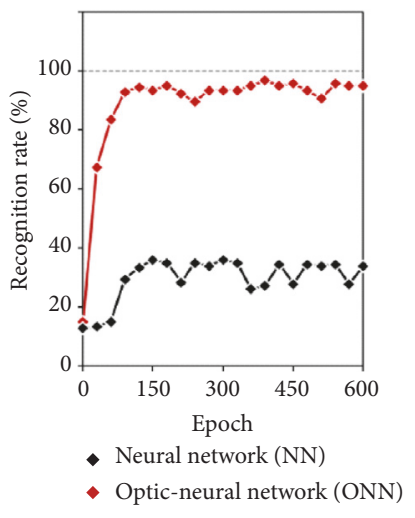

(e)

FIGURE 10: (a) Schematics of device structure and interconnection. (b) Wavelength-dependent conductance change for long-term potentiation and long-term depression. (c) Comparisons of conventional NN and ONN. In conventional NN, the synapses cannot sense the optical information. In ONN, optical sensing functions are added to the synaptic devices, exhibiting wavelength-dependent synaptic weights. (d) Training set and test set for the color-mixed pattern recognition. (e) Comparisons of recognition rate as a function of training epochs in NN and ONN. Reproduced with permission from [64].

integration density in the integrated circuits. To develop appropriate 2D material ORAMs with two-terminal structure can be a potential approach for low-power and highintegration-density devices in the future.

The ORAM synaptic device that integrates optical sensing and synaptic functions have exhibits great potentials in neuromorphic computing and visual information processing. For the future artificial intelligence, to mimic the sensing and processing of auditory or olfactory information like the human brain is also demanded for energy-efficient and miniaturized processing system. As the conventional visual, olfactory and auditory sensors generate a large amount of redundant data, neuromorphic sensory devices potentially allow for the real-time storage and processing of the sensory data with reduced power consumption. Similar to the extension from optical sensors to ORAM synaptic devices, olfactory sensors (e.g., gas sensors) and the auditory sensors (e.g., sound detectors) can also be potentially developed as neuromorphic sensors with synaptic behaviors through appropriate structure design. For both sound detectors and gas sensors, 2D materials have also exhibited their potentials in achieving high sensitivity. More specifically, 2D materials (TMDs, BP, silicene, MXene, etc.) are adopted as promising candidates for high-sensitive gas sensors because of the large surface-to-volume ratio and tunable functionality of the surface through decorating transition metals or functional groups [65-69]. The excellent mechanical properties and piezoelectric properties in $2 \mathrm{D}$ materials also implicate the applications of sound detectors [70-72]. The outstanding performances in $2 \mathrm{D}$ based gas sensors and sound detectors suggest the possibilities for future designs of neuromorphic olfactory sensors and auditory sensors, which could contribute to important applications in the development of edge computing and Internet of Things.

\section{Conflicts of Interest}

The authors declare that they have no conflicts of interest. 


\section{Acknowledgments}

This work was supported by Research Grant of Council of Hong Kong (PolyU 152053/18E), the Hong Kong Polytechnic University (G-YBPS and G-SB79), and National Natural Science Foundation of China (61851402 and 61861166001).

\section{References}

[1] T. Leydecker, M. Herder, E. Pavlica et al., "Flexible nonvolatile optical memory thin-film transistor device with over 256 distinct levels based on an organic bicomponent blend," Nature Nanotechnology, vol. 11, no. 9, pp. 769-775, 2016.

[2] X. Zhu and W. D. Lu, "Optogenetics-inspired tunable synaptic functions in memristors," ACS Nano, vol. 12, no. 2, pp. 12421249, 2018.

[3] F. Zhou, Y. Liu, X. Shen, M. Wang, F. Yuan, and Y. Chai, "Lowvoltage, optoelectronic $\mathrm{CH}_{3} \mathrm{NH}_{3} \mathrm{PbI}_{3-x} \mathrm{Cl}_{x}$ memory with integrated sensing and logic operations," Advanced Functional Materials, vol. 28, no. 15, Article ID 1800080, 2018.

[4] H. Tan, G. Liu, X. Zhu et al., "An optoelectronic resistive switching memory with integrated demodulating and arithmetic functions," Advanced Materials, vol. 27, no. 17, pp. 27972803, 2015.

[5] J. Shim, S. Jo, M. Kim, Y. J. Song, J. Kim, and J. Park, "Lighttriggered ternary device and inverter based on heterojunction of van der waals materials," ACS Nano, vol. 11, no. 6, pp. 63196327, 2017.

[6] Z. Cheng, C. Ríos, W. H. Pernice, C. D. Wright, and H. Bhaskaran, "On-chip photonic synapse," Science Advances, vol. 3, no. 9, Article ID e1700160, 2017.

[7] H. Tan, G. Liu, H. Yang et al., "Light-gated memristor with integrated logic and memory functions," ACS Nano, vol. 11, no. 11, pp. 11298-11305, 2017.

[8] X. Hong, D. J. Loy, P. A. Dananjaya, F. Tan, C. Ng, and W. Lew, "Oxide-based RRAM materials for neuromorphic computing," Journal of Materials Science, vol. 53, no. 12, pp. 8720-8746, 2018.

[9] C. Li, M. Hu, Y. Li et al., "Analogue signal and image processing with large memristor crossbars," Nature Electronics, vol. 1, no. 1, pp. 52-59, 2018.

[10] M. Kimura, T. Shima, and T. Yamashita, "Artificial retina using thin-film photodiode and thin-film transistor," ECS Transactions, vol. 3, no. 8, pp. 325-331, 2006.

[11] S. Choi, S. H. Tan, Z. Li et al., "SiGe epitaxial memory for neuromorphic computing with reproducible high performance based on engineered dislocations," Nature Materials, vol. 17, no. 4, pp. 335-340, 2018.

[12] J. Kang, Z. Yu, L. Wu et al., "Time-dependent variability in RRAM-based analog neuromorphic system for pattern recognition," Electron Devices Meeting (IEDM), pp. 6.4.1-6.4.4, 2017.

[13] Y. Kim, A. Chortos, W. Xu et al., "A bioinspired flexible organic artificial afferent nerve," Science, vol. 360, no. 6392, pp. 9981003, 2018.

[14] G. J. Lee, C. Choi, D. Kim, and Y. M. Song, "Bioinspired artificial eyes: optic components, digital cameras, and visual prostheses," Advanced Functional Materials, vol. 28, no. 24, Article ID 1705202, 2017.

[15] Y. M. Song, Y. Xie, V. Malyarchuk et al., "Digital cameras with designs inspired by the arthropod eye," Nature, vol. 497, no. 7447, pp. 95-99, 2013.
[16] K.-H. Jeong, J. Kim, and L. P. Lee, "Biologically inspired artificial compound eyes," Science, vol. 312, no. 5773, pp. 557-561, 2006.

[17] H. C. Ko, M. P. Stoykovich, J. Song et al., "A hemispherical electronic eye camera based on compressible silicon optoelectronics," Nature, vol. 454, no. 7205, pp. 748-753, 2008.

[18] F. Zhou, Z. Zhou, J. Chen et al., "Optoelectronic resistive random access memory for neuromorphic vision sensors," Nature Nanotechnology, vol. 14, pp. 776-782, 2019.

[19] F. Yan, Z. Wei, X. Wei, Q. Lv, W. Zhu, and K. Wang, “Toward high-performance photodetectors based on 2D materials: strategy on methods," Small Methods, vol. 2, no. 5, Article ID 1700349, 2018.

[20] N. Huo and G. Konstantatos, "Recent progress and future prospects of 2D-based photodetectors," Advanced Materials, vol. 30, no. 51, Article ID e1801164, 2018.

[21] H. Qiao, J. Yuan, Z. Xu et al., "Broadband photodetectors based on graphene- $\mathrm{Bi}_{2} \mathrm{Te}_{3}$ heterostructure," ACS Nano, vol. 9, no. 2, pp. 1886-1894, 2015.

[22] S. Wang, C. Chen, Z. Yu et al., "A $\mathrm{MoS}_{2} / \mathrm{PTCDA}$ hybrid heterojunction synapse with efficient photoelectric dual modulation and versatility ", Advanced Materials, vol. 31, no. 3, Article ID e1806227, 2018.

[23] Q. Guo, A. Pospischil, M. Bhuiyan et al., "Black phosphorus mid-infrared photodetectors with high gain," Nano Letters, vol. 16, no. 7, pp. 4648-4655, 2016.

[24] M. Liu, X. Yin, E. Ulin-Avila et al., "A graphene-based broadband optical modulator," Nature, vol. 474, no. 7349, pp. 64-67, 2011.

[25] A. Di Bartolomeo, G. Luongo, F. Giubileo et al., "Hybrid graphene/silicon Schottky photodiode with intrinsic gating effect," 2D Materials, vol. 4, no. 2, Article ID 025075, 2017.

[26] T. Mueller, F. Xia, M. Freitag, J. Tsang, and P. Avouris, "Role of contacts in graphene transistors: A scanning photocurrent study," Physical Review B: Condensed Matter and Materials Physics, vol. 79, no. 24, Article ID 245430, 2009.

[27] Y. Xu, J. Yuan, L. Fei et al., "Selenium-doped black phosphorus for high-responsivity 2D photodetectors," Small, vol. 12, no. 36, pp. 5000-5007, 2016.

[28] C. Liu, X. Yan, X. Song, S. Ding, D. W. Zhang, and P. Zhou, "A semi-floating gate memory based on van der Waals heterostructures for quasi-non-volatile applications," Nature Nanotechnology, vol. 13, no. 5, pp. 404-410, 2018.

[29] M. Wang, S. Cai, C. Pan et al., "Robust memristors based on layered two-dimensional materials," Nature Electronics, vol. 1, no. 2, pp. 130-136, 2018.

[30] S. Bertolazzi, P. Bondavalli, S. Roche et al., "Nonvolatile memories based on graphene and related 2D materials," Advanced Materials, vol. 31, no. 10, Article ID e1806663, 2019.

[31] X. Wu, R. Ge, P. A. Chen et al., "Thinnest nonvolatile memory based on monolayer h-BN," Advanced Materials, vol. 31, no. 15, Article ID e1806790, 2019.

[32] R. Ge, X. Wu, M. Kim et al., "Atomristor: nonvolatile resistance switching in atomic sheets of transition metal dichalcogenides," Nano Letters, vol. 18, no. 1, pp. 434-441, 2017.

[33] Y. Zhao, Y. Xie, Z. Liu, X. Wang, Y. Chai, and F. Yan, “Twodimensional material membranes: An emerging platform for controllable mass transport applications," Small, vol. 10, no. 22, pp. 4521-4542, 2014.

[34] Y. Zhao, K. Xu, F. Pan, C. Zhou, F. Zhou, and Y. Chai, "Doping, contact and interface engineering of two-dimensional layered transition metal dichalcogenides transistors," Advanced Functional Materials, vol. 27, no. 19, Article ID 1603484, 2017. 
[35] J. Cheng, C. Wang, X. Zou, and L. Liao, "Recent advances in optoelectronic devices based on $2 \mathrm{~d}$ materials and their heterostructures," Advanced Optical Materials, vol. 7, no. 1, Article ID 1800441, 2019.

[36] S. Bertolazzi, D. Krasnozhon, and A. Kis, "Nonvolatile memory cells based on $\mathrm{MoS}_{2}$ /graphene heterostructures," ACS Nano, vol. 7, no. 4, pp. 3246-3252, 2013.

[37] M. S. Choi, G.-H. Lee, Y.-J. Yu et al., "Controlled charge trapping by molybdenum disulphide and graphene in ultrathin heterostructured memory devices," Nature Communications, vol. 4, no. 1624, 2013.

[38] Q. A. Vu, Y. S. Shin, Y. R. Kim et al., "Two-terminal floatinggate memory with van der Waals heterostructures for ultrahigh on/off ratio," Nature Communications, vol. 7, no. 1, article 12725, 2016.

[39] Y. Shi, X. Liang, B. Yuan et al., "Electronic synapses made of layered two-dimensional materials," Nature Electronics, vol. 1, no. 8, pp. 458-465, 2018.

[40] S. Lei, F. Wen, B. Li et al., "Optoelectronic memory using twodimensional materials," Nano Letters, vol. 15, no. 1, pp. 259-265, 2014.

[41] J. Lee, S. Pak, Y. Lee et al., "Monolayer optical memory cells based on artificial trap-mediated charge storage and release," Nature Communications, vol. 8, no. 1, article 14734, 2017.

[42] D. Lee, E. Hwang, Y. Lee et al., "Multibit $\mathrm{MoS}_{2}$ photoelectronic memory with ultrahigh sensitivity," Advanced Materials, vol. 28, no. 41, pp. 9196-9202, 2016.

[43] A. Lipatov, P. Sharma, A. Gruverman, and A. Sinitskii, "Optoelectrical Molybdenum Disulfide $\left(\mathrm{MoS}_{2}\right)$ - Ferroelectric Memories," ACS Nano, vol. 9, no. 8, pp. 8089-8098, 2015.

[44] W. Wang, G. N. Panin, X. Fu et al., " $\mathrm{MoS}_{2}$ memristor with photoresistive switching," Scientific Reports, vol. 6, no. 31224, 2016.

[45] K. Roy, M. Padmanabhan, S. Goswami et al., "Graphene-MoS2 hybrid structures for multifunctional photoresponsive memory devices," Nature Nanotechnology, vol. 8, no. 11, pp. 826-830, 2013.

[46] D. Xiang, T. Liu, J. Xu et al., "Two-dimensional multibit optoelectronic memory with broadband spectrum distinction," Nature Communications, vol. 9, no. 1, article 2966, 2018.

[47] M. D. Tran, H. Kim, J. S. Kim et al., "Two-terminal multibit optical memory via van der waals heterostructure," Advanced Materials, vol. 31, no. 7, Article ID 1807075, 2019.

[48] L. Hu, J. Yuan, Y. Ren et al., "Phosphorene/ZnO nanoheterojunctions for broadband photonic nonvolatile memory applications," Advanced Materials, vol. 30, no. 30, Article ID 1801232, 2018.

[49] Q. Wang, Y. Wen, K. Cai et al., "Nonvolatile infrared memory in MoS2/PbS van der Waals heterostructures," Science Advances, vol. 4, no. 4, Article ID eaap7916, 2018.

[50] Y. Zhai, X. Yang, F. Wang et al., "Infrared-sensitive memory based on direct-grown MoS2-upconversion-nanoparticle heterostructure," Advanced Materials, vol. 30, no. 49, Article ID 1803563, 2018.

[51] A. Bera, H. Peng, J. Lourembam, Y. Shen, X. W. Sun, and T. Wu, "A versatile light-switchable nanorod memory: Wurtzite $\mathrm{ZnO}$ on perovskite SrTiO3," Advanced Functional Materials, vol. 23, no. 39, pp. 4977-4984, 2013.

[52] H. Wang, H. Liu, Q. Zhao et al., "A retina-like dual band organic photosensor array for filter-free near-infrared-tomemory operations," Advanced Materials, vol. 29, no. 32, Article ID 1701772, 2017.
[53] K. Pei, X. Ren, Z. Zhou, Z. Zhang, X. Ji, and P. K. Chan, "A highperformance optical memory array based on inhomogeneity of organic semiconductors," Advanced Materials, vol. 30, no. 13, Article ID 1706647, 2018.

[54] S. Qin, F. Wang, Y. Liu et al., "A light-stimulated synaptic device based on graphene hybrid phototransistor," 2D Materials, vol. 4, no. 3, Article ID 035022, 2017.

[55] J. Jiang, W. Hu, D. Xie et al., "2D electric-double-layer phototransistor for photoelectronic and spatiotemporal hybrid neuromorphic integration," Nanoscale, vol. 11, no. 3, pp. 13601369, 2019.

[56] R. A. John, F. Liu, N. A. Chien et al., "Synergistic gating of electro-iono-photoactive 2D chalcogenide neuristors: coexistence of hebbian and homeostatic synaptic metaplasticity," Advanced Materials, vol. 30, no. 25, Article ID 1800220, 2018.

[57] Z. Ni, Y. Wang, L. Liu et al., "Hybrid structure of silicon nanocrystals and 2D WSe 2 for broadband optoelectronic synaptic devices," IEEE International Electron Devices Meeting (IEDM), pp. 38.5.1-38.5.4, 2018.

[58] H. Tian, X. Wang, F. Wu, Y. Yang, and T.-L. Ren, "High performance 2D perovskite/graphene optical synapses as artificial eyes," IEEE International Electron Devices Meeting (IEDM), pp. 38.6.1-38.6.4, 2018.

[59] K. Pilarczyk, A. Podborska, M. Lis et al., "Synaptic behavior in an optoelectronic device based on semiconductor-nanotube hybrid," Advanced Electronic Materials, vol. 2, no. 6, Article ID 1500471, 2016.

[60] H. K. Li, T. Chen, P. Liu et al., "A light-stimulated synaptic transistor with synaptic plasticity and memory functions based on $\mathrm{InGaZnO}_{x}-\mathrm{Al}_{2} \mathrm{O}_{3}$ thin film structure," Journal of Applied Physics, vol. 119, no. 24, Article ID 244505, 2016.

[61] Y. Wang, Z. Lv, J. Chen et al., "Photonic synapses based on inorganic perovskite quantum dots for neuromorphic computing," Advanced Materials, vol. 30, no. 38, Article ID 1802883, 2018.

[62] S. Gao, G. Liu, H. Yang et al., "An oxide Schottky junction artificial optoelectronic synapse," ACS Nano, vol. 13, no. 2, pp. 2634-2642, 2019.

[63] S. Dai, X. Wu, D. Liu et al., "Light-stimulated synaptic devices utilizing interfacial effect of organic field-effect transistors," ACS Applied Materials \& Interfaces, vol. 10, no. 25, pp. 21472-21480, 2018.

[64] S. Seo, S.-H. Jo, S. Kim et al., "Artificial optic-neural synapse for colored and color-mixed pattern recognition," Nature Communications, vol. 9, no. 1, article 5106, 2018.

[65] J. Park, J. Mun, J.-S. Shin, and S.-W. Kang, "Highly sensitive twodimensional $\mathrm{MoS}_{2}$ gas sensor decorated with Pt nanoparticles," Royal Society Open Science, vol. 5, no. 12, Article ID 181462, 2018.

[66] S. Cui, H. Pu, and S. A. Wells, "Ultrahigh sensitivity and layer-dependent sensing performance of phosphorene-based gas sensors," Nature Communications, vol. article, no. 8632, 2015.

[67] X.-P. Chen, L.-M. Wang, X. Sun et al., "Sulfur dioxide and nitrogen dioxide gas sensor based on arsenene: a first-principle study," IEEE Electron Device Letters, vol. 38, no. 5, pp. 661-664, 2017.

[68] V. Q. Bui, T. Pham, D. A. Le, C. M. Thi, and H. M. Le, "A first-principles investigation of various gas $(\mathrm{CO}, \mathrm{H} 2 \mathrm{O}, \mathrm{NO}$, and O2) absorptions on a WS2 monolayer: stability and electronic properties," Journal of Physics: Condensed Matter, vol. 27, no. 30, Article ID 305005, 2015.

[69] A. N. Abbas, B. Liu, L. Chen et al., "Black phosphorus gas sensors," ACS Nano, vol. 9, no. 5, pp. 5618-5624, 2015. 
[70] L.-Q. Tao, H. Tian, Y. Liu et al., "An intelligent artificial throat with sound-sensing ability based on laser induced graphene," Nature Communications, vol. 8, no. 1, article 14579, 2017.

[71] R. Hinchet, U. Khan, C. Falconi, and S.-W. Kim, "Piezoelectric properties in two-dimensional materials: Simulations and experiments," Materials Today, vol. 21, no. 6, pp. 611-630, 2018.

[72] C. Cui, F. Xue, W. Hu, and L. Li, "Two-dimensional materials with piezoelectric and ferroelectric functionalities," $n p j 2 D$ Materials and Applications, vol. 2, no. 1, article 18, 2018. 